To appear in ApJ

\title{
Spitzer, Near-Infrared, and Submillimeter Imaging of the Relatively Sparse Young Cluster, Lynds 988e
}

\author{
Thomas S. Allen ${ }^{1}$, Judith L. Pipher ${ }^{1,2}$, Robert A. Gutermuth ${ }^{3,2}$, S. Thomas Megeath ${ }^{4,2}$, \\ Joseph D. Adams ${ }^{5}$, Terry L. Herter ${ }^{5}$, Jonathan P. Williams ${ }^{6}$, Jennifer A. Goetz-Bixby ${ }^{1}$, \\ Lori E. Allen ${ }^{3}$, Philip C. Myers ${ }^{3}$
}

\begin{abstract}
We present Spitzer images of the relatively sparse, low luminosity young cluster L988e, as well as complementary near-infrared (NIR) and submillimeter images of the region. The cluster is asymmetric, with the western region of the cluster embedded within the molecular cloud, and the slightly less dense eastern region to the east of, and on the edge of, the molecular cloud. With these data, as well as with extant $\mathrm{H} \alpha$ data of stars primarily found in the eastern region of the cluster, and a molecular ${ }^{13} \mathrm{CO}$ gas emission map of the entire region, we investigate the distribution of forming young stars with respect to the cloud material, concentrating particularly on the differences and similarities between the exposed and embedded regions of the cluster. We also compare star formation in this region to that in denser, more luminous and more massive clusters already investigated in our comprehensive multi-wavelength study of young clusters within $1 \mathrm{kpc}$ of the Sun.
\end{abstract}

Subject headings: pre-main sequence — stars: formation — infrared: stars

\footnotetext{
${ }^{1}$ Department of Physics and Astronomy, University of Rochester, Rochester, NY 14627

${ }^{2}$ Visiting Astronomer, Kitt Peak National Observatory, National Optical Astronomy Observatory, which is operated by the Association of Universities for Research in Astronomy, Inc. (AURA) under cooperative agreement with the National Science Foundation.

${ }^{3}$ Harvard-Smithsonian Center for Astrophysics, Mail Stop 42, 60 Garden Street, Cambridge, MA 02138

${ }^{4}$ University of Toledo, Department of Physics and Astronomy, Toledo OH 43606

${ }^{5}$ Cornell University, Department of Radiophysics Space Research, Ithaca NY 14853

${ }^{6}$ Institute for Astronomy, University of Hawaii, 2680 Woodlawn Drive, Honolulu, HI 96822
} 


\section{INTRODUCTION}

Lynds 988e (L988e =IRAS 21023+5002) was identified as one of six IRAS point sources (L988a-f) observed toward the L988 dark cloud, which is located on the edge of the Cygnus OB7 molecular cloud association (Dobashi et al. 1994) and which contains several bright, pre-main sequence objects. Bipolar molecular outflows were discovered (Clark 1986) to be associated with the vicinity of L988e, as well as the vicinity of L988a and L988f, and there is also high velocity $\mathrm{CO}$ emission (blue-shifted lobe only) to the west of L988c. There are two $\mathrm{LkH} \alpha$ objects and related nebulosity seen both at visible and infrared wavelengths, $\mathrm{LkH} \alpha$ $324-\mathrm{SE}$ and $\mathrm{LkH} \alpha 324$, the brightest cluster members of a region of $\sim 50 \mathrm{H} \alpha$ emission-line stars (Herbig \& Dahm 2006) on the edge of the heavy extinction associated with L988e's parent molecular cloud. Most of these emission-line stars lie to the east of the molecular cloud or on its eastern edge. $\mathrm{LkH} \alpha 324$ is a Herbig Ae/Be (HAeBe) star, close (in projection) to the IRAS coordinate, and $\mathrm{LkH} \alpha 324-\mathrm{SE}$ (misidentified as $\mathrm{LkH} \alpha 324$ in the the Herbig and Bell catalog (Herbig \& Bell 1988)) is probably also an HAeBe star (Herbig \& Dahm 2006). Both Classical T Tauri stars (CTTS) and Weak-line T Tauri stars (WTTS) are included in the emission-line stellar population. Employing an average extinction of $\mathrm{A}_{V} \sim 2.5 \mathrm{mag}$ for most of the population, and measured extinctions for a few objects, Herbig \& Dahm (2006) estimate median ages of 0.6 and 1.7 Myr for the emission-line stellar population from $V$ and $I$ photometry: for these estimates they assume evolutionary isochrones from D'Antona \& Mazzitelli (1997), and Siess et al. (2000) respectively. In this paper, we examine properties of the entire cluster, including both the emission-line stellar population and an obscured population to the west within the molecular cloud which we have identified here, and refer to as the embedded population.

At a distance of $\sim 700 \mathrm{pc}$ and a far infrared luminosity of $\sim 200 L_{\odot}$, the cluster of young pre-main sequence stars associated with L988e is relatively sparse, and in the bottom quartile in far-infrared luminosity of the northern young cluster sample surveyed in ${ }^{13} \mathrm{CO}$ and $\mathrm{C}^{18} \mathrm{O}$ molecular gas (Ridge et al. 2003) (see Table 1). Despite present interest in young cluster evolution as discussed, for example, in a recent review (Lada \& Lada 2003), with the exception of papers by Hodapp (1994) and Herbig \& Dahm (2006), the young cluster associated with L988e has been relatively unstudied at infrared wavelengths to date. Hereafter we refer to the young cluster as the L988e cluster, or just L988e.

In this paper, we utilize mid-infrared (MIR) images of L988e obtained with the Infrared Array Camera (IRAC) and $24 \mu \mathrm{m}$ images obtained with the Multi-Band Imaging Photometer (MIPS) on the Spitzer Space Telescope1 (see a composite three-color Spitzer image of

\footnotetext{
${ }^{1}$ This work is based [in part] on observations made with the Spitzer Space Telescope, which is operated
} 
the L988e region, also including L988f and c in Fig. 1, also see Fig. 2 where the various fields of view are shown on a reproduction of the POSS V-band image of the region), as well as complementary near-infrared (NIR) ground-based images, to identify cluster members via their infrared excess emission from protostellar envelopes, and from circumstellar disks. The spatial distribution of identified protostars and disk objects in L988e is compared with the $850 \mu \mathrm{m}$ continuum maps of cold dust emission obtained with Submillimeter Common-User Bolometer Array (SCUBA) on the James Clerk Maxwell Telescope (JCMT), with dust extinction maps generated using NIR data, and with a ${ }^{13} \mathrm{CO}$ map of the associated molecular clouds (Ridge et al. 2003). These data were gathered as part of a coordinated multi-wavelength Young Stellar Cluster Survey, with the goal of providing the spatial distribution of a complete census of YSOs - young stellar objects (all objects with an infrared excess, including stars with disks and protostars) in 30 nearby embedded clusters chosen from a list of 73 young groups and clusters within $1 \mathrm{kpc}$ of the Sun (Porras et al. 2003). Program clusters are representative of quite diverse star-forming regions which vary in number of cluster members, member number density, luminosity, and environment. This coordinated study, which includes NIR, mid-infrared (MIR), submillimeter and millimeter CO imaging of the regions, is designed to advance our understanding of clustered star formation. A comprehensive paper examining YSO spatial distributions in this survey is in preparation: in a few instances, where unique data and/or characteristics distinguish the object, a separate paper is written. Here, the availability of deep NIR images and a published catalog of $\mathrm{H} \alpha$ emission-line stars (Herbig \& Dahm 2006) distinguishes this object from the others in the survey.

Several authors have described efforts to define IRAC-only (Allen et al. 2004), or combined IRAC/MIPS (Muzerolle et al. 2004) protocols which differentiate between stars with dusty circumstellar accretion disks (class II), and protostars or objects dominated by dust emission from envelopes (class I). All class I and II objects exhibit infrared excess over photospheric emission typical of more evolved young cluster members (diskless or optically thin disk class III) as well as of foreground/background normal stars. Since substantial and/or variable interstellar extinction can cause ambiguity in identification of these objects using IRAC-only methods (Gutermuth 2005), and the MIPS $24 \mu \mathrm{m}$ images may not detect the faintest YSOs, a more robust classification method is required. Gutermuth (2005) used seven-band photometry from $J$-band to IRAC $8.0 \mu \mathrm{m}$ to systematically determine dereddened SED slopes. Using this classification as a standard, methods requiring five-band detection at $J(1.25 \mu \mathrm{m}), H(1.65 \mu \mathrm{m}), K_{s}(2.15 \mu \mathrm{m}),[3.6](3.6 \mu \mathrm{m}),[4.5](4.5 \mu \mathrm{m})$, or four-band detection at $H, K_{s},[3.6],[4.5]$ also have been demonstrated in the same publica-

by the Jet Propulsion Laboratory, California Institute of Technology under a contract with NASA. 
tion. Here we primarily employ a combination of the five-band and four-band methods, but we also use slopes deduced from IRAC photometry in combination with MIPS $24 \mu \mathrm{m}$ photometry, where available, to classify protostars and transition disk objects (objects with a pronounced inner disk gap) in L988e. Alternate methods are exploited for the young cluster

IC 348 (Lada et al. 2006): they utilize a simple power-law fit to the observed spectral energy distribution (SED) from the 4 IRAC bands to determine a slope $\alpha_{I R A C}$, and as well study the SED from visual to far-infrared wavelengths for all cluster members as a function of spectral class.

\section{OBSERVATIONS \& DATA ANALYSIS}

\subsection{Near-Infrared Data}

NIR images in the $J, H$, and $K_{s}$ wavebands of L988e were first obtained by the authors on 2002 October 26 using the Simultaneous Quad Infrared Imaging Device (SQIID), which houses four $1024 \times 1024$ InSb arrays, of which only $512 \times 512$ are used, on the $2.1-\mathrm{m}$ telescope at the Kitt Peak National Observatory, a facility of the National Optical Astronomical Observatory. The plate-scale of SQIID on the $2.1-\mathrm{m}$ is $0^{\prime \prime} .68 \mathrm{pixel}^{-1}$, and the circular field of view is $5^{\prime} .8$ in diameter. SQIID images, obtained in an overlapping $3 \times 3$ grid pattern, were used to construct a mosaic comparable in size to the $15^{\prime} \times 15^{\prime}$ Spitzer IRAC field of view. Twelve dithered mosaics were obtained in each band with a total exposure time of $60 \mathrm{~s}$ per image at $J, H$, and $K_{s}$, leading to $720 \mathrm{~s}$ of exposure time. With seeing, the typical point spread function (PSF) was $1^{\prime \prime} .9 \mathrm{FWHM}$ at $J$-band and $1^{\prime \prime} .77$ in the other two bands, and the $90 \%$ integrated completeness limits of the final mosaics were estimated (Gutermuth et al. 2005) as $K_{s}=16.19, H=16.20$, and $J=17.71$. However, the image quality suffered because all images exhibit position-dependent coma. PSF photometry was consequently impossible, and aperture photometry with large apertures was impractical because of the crowded field.

Higher spatial resolution, deep, NIR observations in the $J(1.25 \mu \mathrm{m}), H(1.65 \mu \mathrm{m})$, and $K_{s}(2.15 \mu \mathrm{m})$ wavebands were obtained on 2004 July 6 with the Palomar Wide-field IR Camera (WIRC) which houses a $2048 \times 2048 \mathrm{HgCdTe}$ array (Wilson et al. 2003). The platescale of WIRC is $0^{\prime \prime} .249$ pixel $^{-1}$, and the field of view is $8^{\prime} .7$ on a side. In addition, the PSF was $1^{\prime \prime} .1$ in the $K_{s}$ band and $1^{\prime \prime} .3$ in the other two bands. The observing sequence consisted of a large ( $3^{\prime} .5$ offset) five-point dither pattern. The $90 \%$ integrated photometric completeness limits of the final mosaics were estimated (Gutermuth et al. 2005) to be approximately $K_{s}=$ $17.21, H=17.72$, and $J=18.22$.

Basic reduction of the SQIID NIR data was performed at Rochester using custom IDL 
routines, developed by the authors, which include modules for linearization, flat-field creation and application, background frame creation and subtraction, distortion measurement and correction, and mosaicking. Point source detection and synthetic aperture photometry of all point sources were carried out using PhotVis version 1.09, an IDL GUI-based photometry visualization tool (Gutermuth et al. 2004). By visual inspection, those detections that were identified as structured nebulosity were considered non-stellar and rejected. Aperture photometry was performed using radii of $2^{\prime \prime} .04,3^{\prime \prime} .40$, and $5^{\prime \prime} .44$ for the aperture, inner, and outer sky annular limits, respectively.

WIRC data reduction was completed at Cornell using custom IDL routines and inhouse image processing software. Processing steps included dark subtraction, linearization, sky subtraction, flat-fielding, spatial flux gradient correction (due to a dependency of the photometric zero point on position on the array; Jarrett personal comm.), and mosaicking. Aperture photometry using APPHOT was applied to all point sources. Radii of the apertures and inner and outer limits of the sky annuli were $1^{\prime \prime} .5,2^{\prime \prime} .75$, and $3^{\prime \prime} .5$ respectively. PSF fitting was attempted, but the PSF varied sharply across the images, with resulting residuals larger than obtained for the aperture photometry discussed above. Aperture photometry with larger apertures was tested, but not applied because it failed in the crowded field. WIRC photometry was calibrated by minimizing residuals to corresponding 2MASS detections for selected stars with magnitudes between $\sim 12-14$ mag. Outliers were rejected from the fit (outliers are confused or unresolved multiples in the 2MASS dataset). The rms scatter for the $J, H$, and $K_{s}$ waveband residuals are 0.06, 0.07, and 0.07 mag respectively. Carpenter (2001) showed that $\sim 7 \%$ of YSOs toward Orion A are variable: variability may contribute to the observed scatter. Photometric magnitudes for stars which were brighter than the range of magnitudes corrected by the linearization modules were replaced with 2MASS photometry. Because of the superior spatial resolution, depth, image quality, yet similar field of view of the WIRC dataset, we use it for classification for all sources in L988e, and include photometry in Tables 2, 3, 4, and 5, The SQIID dataset was used only for internal verification, and is not tabulated here. For fainter magnitudes (14-16) after rejecting outliers beyond $3 \sigma$, the rms scatter of the $J, H$, and $K_{s}$ waveband residuals between the SQIID and WIRC datasets was $0.08,0.11$, and $0.11 \mathrm{mag}$ respectively. We believe the WIRC photometry to be more accurate for the reasons cited above.

Fig. 3 shows a three-color (WIRC $J, H, K_{s}$ ) image of the region with ${ }^{13} \mathrm{CO}$ line emission contours (Ridge et al. 2003) overplotted. Visual inspection of the figure shows that the stellar density to the east of the molecular cloud is considerably higher than within the extent of the molecular cloud, as expected. 


\subsection{Mid-Infrared Data}

Mid-infrared images were obtained with Spitzer/IRAC at 3.6, 4.5, 5.8, and $8.0 \mu \mathrm{m}$ and Spitzer/MIPS at $24 \mu \mathrm{m}$ as part of the Spitzer Young Stellar Cluster Survey. The IRAC images were obtained 2004 July 27, and each image was $5^{\prime}$ on a side with $1^{\prime \prime} .2$ pixels. They were taken in $12 \mathrm{~s}$ High Dynamic Range (HDR) mode, whereby a $0.4 \mathrm{~s}$ integration is taken, immediately followed by a $10.4 \mathrm{~s}$ integration at the same pointing. Four dithered $3 \times 4$ mosaics were obtained at each wavelength. The typical PSF is approximately $2^{\prime \prime} .2$ at 3.6 and $4.5 \mu \mathrm{m}, 2^{\prime \prime} .3$ at $5.8 \mu \mathrm{m}$, and $2^{\prime \prime} .5$ at $8.0 \mu \mathrm{m}$. Ninety percent completeness limits of the final mosaics are approximately $[3.6]=14.68,[4.5]=14.67,[5.8]=13.72$, and $[8.0]=12.73$, and were evaluated using the same estimation technique as used for the NIR data. The Spitzer/MIPS $24 \mu \mathrm{m}$ images were obtained on 2003 December 29, using scan mode at a medium scan rate. The total area imaged is about $20^{\prime} \times 1^{\circ}$. The typical point spread function is approximately $6^{\prime \prime} .1$, and the $90 \%$ completeness limit is $[24]=8.18$.

Basic reduction of the Spitzer/IRAC data was executed using the Spitzer Science Center's Basic Calibrated Data (BCD) pipelines and Spitzer/MIPS data using the Post-BCD product. The IRAC and MIPS Data handbook 2 give complete descriptions of the calibration processes for point sources. The IRAC Data Handbook performs calibration photometry on a set of isolated standard stars using a 10 pixel $\left(12^{\prime \prime}\right)$ aperture, and 10 and 20 pixel radii of the sky annulus, but notes that considerably smaller apertures are required in crowded fields, and that small off-source sky annuli are needed when the point sources are embedded in extended emission. In those cases, aperture corrections are required, and the Data Handbook 3.0 provides aperture corrections for various standard aperture and annuli configurations for pipeline version S13. The same considerations apply to the MIPS data.

Post-pipeline processing of IRAC data was performed at Rochester using custom IDL routines, developed by the authors, which include modules for bright source artifact correction, cosmic ray removal and mosaicking. Point source extraction and aperture photometry were performed using PhotVis version 1.09. Radii of $2^{\prime \prime} .4,2^{\prime \prime} .4$, and $7^{\prime \prime} .2$ were used for the aperture, inner, and outer sky annular limits, respectively, for the IRAC data and radii of $7^{\prime \prime} .4,14^{\prime \prime} .7$, and $24^{\prime \prime} .5$ were used for the aperture, inner and outer sky annular limits, respectively, for the MIPS data. For a Gaussian PSF, an aperture radius equal to the FWHM contains $93.7 \%$ of the total flux. Increasing this aperture would negligibly gain signal from the source and increases total noise from the substantial MIR background emission. The two short-wave IRAC detector channels, 3.6 and $4.5 \mu \mathrm{m}$ respectively, have approximately

${ }^{2}$ The IRAC and MIPS Data Handbooks can be found at http://ssc.spitzer.caltech.edu/irac/dh/ and http://ssc.spitzer.caltech.edu/mips/dh/, respectively. 
Gaussian PSFs (other than the effects of diffraction), so that choosing an aperture radius approximately equal to the observed FWHM implies only a small correction because of the small aperture. Point source images for the two long-wave IRAC detector channels, 5.8 and $8.0 \mu \mathrm{m}$ respectively, do not have a Gaussian PSF: much of the point source intensity is scattered in bands along rows and columns, as well as over the entire array and beyond (see Data Handbook and Pipher et al. (2004)), but is well-characterized (see Reach et al. (2005)). The chosen aperture of $2^{\prime \prime} .4$ only gives $63 \%$ of the source flux from a 12 " aperture in the 8.0 $\mu \mathrm{m}$ band. In this band, PAH emission nebulosity is very bright and often structured, necessitating use of relatively small apertures and sky annuli. IRAC photometry was calibrated using zero-magnitude flux densities of $280.9 \pm 4.1,179.7 \pm 2.6,115.0 \pm 1.7$, and $64.13 \pm 0.94$ Jy in the 3.6, 4.5, 5.8, and $8.0 \mu \mathrm{m}$ bands respectively (Reach et al. 2005) in a 10 pixel $\left(12^{\prime \prime}\right)$ aperture, and aperture correction factors of $1.213,1.234,1.578$, and 1.584 respectively as tabulated in the Data Handbook. MIPS photometry was calibrated using a zero-point flux of 7.3 Jy, referenced to Vega (MIPS Data Handbook 2005).

\subsection{Submillimeter Data}

L988e was observed with the SCUBA submillimeter camera at $850 \mu \mathrm{m}$ on the James Clerk Maxwell Telescope 3 (JCMT) on Mauna Kea, Hawaii over the course of 5 nights from March to August 2003 in relatively poor weather conditions $(\tau(225 \mathrm{GHz})=0.25-0.5)$. The data were obtained in scan-mapping mode whereby a map is built up by sweeping the telescope across the source while chopping the secondary at a series of throws, $30^{\prime \prime}, 44^{\prime \prime}$, and $68^{\prime \prime}$. To minimize striping artifacts, each set of three sweeps was performed alternately in right ascension and declination. The SURF reduction package (Jenness et al. 1998) was used to flat-field the data, remove bad pixels, and make images. Although effective at removing the high sky and instrument background, this observing technique necessarily loses all features on scales larger than the largest chopper throw, $68^{\prime \prime}$. The calibration was performed by using the same observing technique to observe one or more of Mars, Uranus, and standard source CRL 2688. Fig. 4 presents the SCUBA map in gray-scale, with ${ }^{13} \mathrm{CO}$ contours and YSOs (see below) overplotted. This map is presented here for morphological comparison to cluster member spatial distributions.

\footnotetext{
${ }^{3}$ The James Clerk Maxwell Telescope is operated by The Joint Astronomy Centre on behalf of the Particle Physics and Astronomy Research Council of the United Kingdom, the Netherlands Organisation for Scientific Research, and the National Research Council of Canada.
} 


\subsection{Extinction Maps}

We derive the $\mathrm{A}_{K s}$ extinction maps using the $\left(H-K_{s}\right)$ colors of all stars $(11,313)$ detected in both bandpasses, using a method outlined in more detail by Gutermuth et al. (2005). Briefly, the line of sight extinction to each point on a $2^{\prime \prime}$ grid throughout the region surrounding the L988e core was estimated using a variation of the nearest neighbor technique. At each point the mean and standard deviation of the $\left(H-K_{s}\right)$ colors of the $N=20$ nearest stars to each grid point were calculated, using an iterative outlier rejection algorithm that would reject any star with an $\left(H-K_{s}\right)$ color $>3 \sigma$ from the mean, until the mean converged. The assumed NIR portion of the reddening law (Flaherty et al. 2007) given in Table 6 was used to convert the $\left(H-K_{s}\right)$ value at each point to $\mathrm{A}_{K s}$, using $\mathrm{A}_{K s}=1.82\left(\left(H-K_{s}\right)\right)_{o b s}$ $\left.\left.\left(H-K_{s}\right)\right)_{\text {int }}\right)$, where $\left(H-K_{s}\right)_{\text {int }}=0.2$ is assumed as an average intrinsic $\left(H-K_{s}\right)$ color for stars in young clusters. Further, the $H-K_{s}$ map was convolved with a $15^{\prime \prime}$ Gaussian kernel to match the SCUBA beam size. We compare the resultant map of $\mathrm{A}_{K s}$ with the ${ }^{13} \mathrm{CO}$ map from Ridge et al. (2003) in Fig. 5. As can be seen, the WIRC-derived $\mathrm{A}_{K s}$ extinction map follows the general distribution of the molecular cloud as outlined by CO emission, but provides considerably greater spatial detail and extent.

If stars within the molecular cloud are included in the above analysis, a biased estimation of $\mathrm{A}_{K s}$ could result. In regions where cluster members dominate the $N=20$ number counts, this estimation will be lower than the line of sight extinction through the entire cloud, and thsi deviation will be larger in regions of high extinction where stars beyond the cloud cannot be detected. To account for this bias, the $\left(H-K_{s}\right)$ derived extinction map has been augmented with an extinction map derived by estimating $\mathrm{A}_{K s}$ using SCUBA $850 \mu \mathrm{m}$ flux, and utilizing a conversion factor of $\mathrm{A}_{K_{s}} / \mathrm{F}_{850}=0.272 \mathrm{mag}$ pixel $\mathrm{mJy}^{-1}$ and an assumed

dust temperature of $30 \mathrm{~K}$ (Gutermuth et al. 2005). In areas where the SCUBA derived $\mathrm{A}_{K s}$ values exceed the $\left(H-K_{s}\right)$ - derived $\mathrm{A}_{K s}$ values by $30 \%$, the SCUBA derived values are used. Only one small region coincident with the L988e molecular core required augmentation with SCUBA-derived extinction values in the WIRC extinction map.

\section{IDENTIFYING YOUNG STELLAR OBJECTS}

\subsection{NIR and MIR YSO identification Methods}

Gutermuth (2005) has shown that by de-reddening the NIR/IRAC colors of young cluster stars, the IRAC-only classification scheme (Allen et al. 2004) can be modified to better isolate class II from class I objects. Briefly, Gutermuth (2005) reevaluated the original IRAC-only classification scheme by considering the SED slopes from the NIR to the MIR. 
Slopes $\left(\alpha=\mathrm{d} \log \left(\lambda F_{\lambda}\right) / \mathrm{d} \log (\lambda)\right)$ were constructed for both measured photometry and de- reddened photometry of point sources in the cluster field of view. When de-reddened photometry was utilized on six clusters, histograms of numbers of stars vs. 2-8 $\mu \mathrm{m}$ SED slope separate into 3 bins corresponding to field stars or class III $(\alpha<-2)$, class II $(-2<$ $\alpha<-0.5)$, and class I $(\alpha>-0.5)$, (see Fig. 6). This figure is constructed using the reddening law discussed above (Flaherty et al. 2007), derived from IRAC and NIR cluster data: this reddening law proved measurably different from a previously published reddening law utilizing IRAC and NIR data (Indebetouw et al. 2005) that is primarily associated with reddening from dust in the diffuse interstellar medium rather than from dust in a molecular cloud.

In addition, Gutermuth (2005) considered classification methods using $J, H, K_{s},[3.6],[4.5]$ photometry as well as $H, K_{s},[3.6]$, [4.5] photometry. Where $J$-band detections are available, objects are de-reddened to the $(J-H)_{o}$ vs. $\left(H-K_{s}\right)_{o}$ CTTS locus of Mever et al. (1997) in the former method, or lacking a $J$-band detection, to the $\left(H-K_{s}\right)_{o}$ vs. $([3.6]-[4.5])_{o}$ CTTS locus from Gutermuth (2005) in the latter. Gutermuth (2005) then applied the seven-band classifications to a de-reddened $(K-[3.6])_{o}$ vs. $([3.6]-[4.5])_{o}$ color-color diagram, where the class I and II objects proved to be well separated.

Since the 5.8 and $8.0 \mu \mathrm{m}$ photometry is less sensitive, we utilize de-reddened (see Table6 for the assumed reddening law for young clusters) $(J), H, K_{s}$, [3.6], [4.5] photometry to classify most objects via their location on the $(K-[3.6])_{o}$ vs. $([3.6]-[4.5])_{o}$ color-color diagram: these classifications are color-coded in Fig. 7. Where $24 \mu \mathrm{m}$ data are available, objects classified as class I that have $([5.8]-[24])<4$ were reclassified as class II. Additional class I and class II objects (hereafter class Is and class IIs, similarly class IIIs for class III objects) were identified utilizing SED slopes from IRAC and MIPS photometry, even when some objects were not detected in the $H, K_{s}$, or [3.6] bands. In addition, there are a handful of objects with NIR+IRAC colors of class IIIs, but which exhibit a $24 \mu \mathrm{m}$ excess (e.g. $([5.8]-[24])>1.5$ and $([8]-[24])>1.5)$ : these objects are classified as transition disk objects, YSOs characterized by a cool circumstellar disk containing a substantial inner gap. However, it must be noted that in the absence of a large $24 \mu \mathrm{m}$ excess, it is difficult to distinguish primordial transition disk objects (with ages less than a few Myr) from regenerative debris disk objects (with ages of tens of Myr to a few Gyr). Since the field of view covered by MIPS (as well as [3.6] and [5.8]) extends farther to the west than the $(J), H, K_{s}$, [3.6], [4.5] field, additional YSOs are identified outside that field. 


\subsection{Isolating Extragalactic Contamination}

Deep NIR and MIR photometric detections of YSOs toward molecular clouds are contaminated by detection of extragalactic objects, including PAH (polycyclic aromatic hydrocarbon)-rich galaxies and active galactic nuclei (AGN), both of which have colors similar to YSOs. We account for extragalactic contamination in the following way. For objects which have detections in all four of the IRAC bands, we deredden each object on the assumption that it lies behind the molecular cloud, utilizing the extinction map discussed in $§ 2.4$. We then utilize $([4.5]-[5.8])$ vs. $([5.8]-[8.0])$ and $([3.6]-[5.8])$ vs. $([4.5]-[8.0])$ colorcolor diagrams to identify the PAH-rich galaxy contaminants and the $[4.5]$ vs. ([4.5] $-[8.0])$ color-magnitude diagram to identify the AGN contaminants. The prescriptions for identification are described in detail by Gutermuth et al. (2007). For objects which only have $(J) H K[3.6][4.5]$ photometry, we are unable to directly use those prescriptions to identify extragalactic contaminants. Instead, inspection of the histogram of de-reddened [3.6] magnitudes for the objects with an infrared excess utilizing the $(J) H K[3.6][4.5]$ classification scheme (Fig. 8) shows a bifurcation in the distribution at [3.6] $=13.5$. Objects with [3.6] $<13.5$ are considered as potential YSOs, while objects with [3.6] $>13.5$ are considered as potential extragalactic contaminants and are rejected as potential YSOs. This scheme is in agreement with previous work stating that at magnitudes fainter than about 14, extragalactic objects dominate number counts (Fazio et al. 2004). In order to quantify this further, we examine the differential number counts for sources detected in the Boötes region in the Flamingos Extragalactic Survey (Elston et al. 2006). At [3.6] = 13.5 (Fig. 8), and a mean dereddened $\left(K_{s}-[3.6]\right)_{o}$ color of 0.79 (typical of YSOs), this bifurcation magnitude corresponds to $K_{s}=14.29$. From Fig. 8 in Elston et al. (2006), we estimate that there are approximately 10 extragalactic sources within our field of view at $K_{s}=14.29$, and the number rapidly increases to fainter magnitudes. Since we are examining the spatial distribution as well as the disk fraction of the L988e population of YSOs, both in-cloud and off-cloud, we want to minimize the possibility of contamination by extragalactic objects. An examination of the $K_{s}$ vs. $\left(H-K_{s}\right)$ color-magnitude diagram (Fig. 9) shows that even excluding $K_{s} \geq 14.29$, we are still capable of detecting relatively low mass YSOs. Faint cluster members (below the hydrogen burning limit at $\mathrm{A}_{V}=0$ ) will also be excluded by these criteria: therefore brown dwarf cluster members will not be considered further. Spectroscopy is the best way to make firm brown dwarf identifications, and beyond the scope of this paper. 


\subsection{Number Counts of Identified YSOs}

Before removing extragalactic contaminants, there are a total of 2293 objects in a $14.7^{\prime} \times$ $12.5^{\prime}$ field with detections in all four bands $\left(H, K_{s},[3.6],[4.5]\right)$ meeting the criterion that the photometric uncertainties are less than $0.1 \mathrm{mag}$ in all bandpasses. Following application of the extragalactic contamination criteria noted above, there remain a total of 678 objects in the NIR + IRAC dataset: these objects will be discussed in the following sections. Of these, three are class Is, 69 class IIs, seven are transition disk objects, and 599 are class IIIs or field stars with no obvious infrared excess. When SED slopes were computed for objects in the same field of view with only IRAC and/or MIPS detections, an additional five class Is, three class IIs, and 36 class IIIs were found. Further, there is one object detected in only the 4.5, 5.8, 8.0, and $24 \mu \mathrm{m}$ wavebands. From the $4.5-24 \mu \mathrm{m}$ SED slope for this object, $\alpha=0.98$, we characterize it as class I. As discussed earlier, a total of six transition disk objects are also classified based on their ([5.8] - [24]) or ([8] - [24]) colors. In Fig. 10 we present a three-color IRAC image ([3.6], [4.5], [8.0]) of the L988e region, with WIRC + IRAC class I and II classifications overplotted, and in Tables 2, 3, 4, and 5 we present a catalog of identified objects.

The MIPS field of view extends far to the northwest of L988e, to include L988c, as noted above. Fortunately, IRAC data in two of the four bands (3.6 and $5.8 \mu \mathrm{m})$ were obtained to the northwest of L988e. From 3-24 $\mu \mathrm{m}$ SED slopes in combination with NIR data, a total of 19 class Is, 73 class IIs, and 643 class IIIs/field stars were found in the $14.7^{\prime} \times 19.4^{\prime}$ field. A three-color IRAC + MIPS image ([3.6], [5.8], [24]) of the extended L988e region was presented in Fig. 1.

\section{THE H $\alpha$ EMISSION LINE PRE-MAIN SEQUENCE POPULATION}

Early studies of the emission-line stellar populations in molecular clouds led to the

classical T Tauri star (CTTS) and the weak T Tauri star (WTTS) definitions (Herbig \& Bell 1988) in terms of their $\mathrm{H} \alpha$ equivalent widths EW[H $\alpha]$. Although the WTTSs usually show weaker $\mathrm{H} \alpha$ emission, limited both in level and profile width (from chromospheric emission), CTTSs exhibit strong emission lines, with broad profiles, generated in the accretion process. By 1988, the CTTS classification was taken to be $\mathrm{EW}[\mathrm{H} \alpha] \geq 10 \AA$. However, in a recent study of low mass stars, White \& Basri (2003) suggest an empirical relationship for the critical $\mathrm{EW}[\mathrm{H} \alpha]$ value as a function of spectral type for low mass stars. Using the presence of optical veiling to identify accretion, they propose the following improved scheme of CTTS classification for spectral classes later than $\mathrm{K} 0$ : $\mathrm{EW}[\mathrm{H} \alpha] \geq 3 \AA$, for K0-K5; $\mathrm{EW}[\mathrm{H} \alpha] \geq$ $10 \AA$, for $\mathrm{K} 7-\mathrm{M} 2.5 ; \mathrm{EW}[\mathrm{H} \alpha] \geq 20 \AA$, for $\mathrm{M} 3-\mathrm{M} 5.5$; and $\mathrm{EW}[\mathrm{H} \alpha] \geq 40 \AA$, for M6-M7.5. 
In what follows, we first use the single equivalent width classification of CTTS/WTTS, and then examine whether this analysis would change appreciably with the White \& Basri (2003) modification.

In Fig. 5, cluster stars, including the HAeBe emission-line stars in this cluster identified by Herbig \& Dahm (2006), are overplotted on an image of the extinction at $K_{s}$-band (see $\S 2.4)$. Each object in Fig. 5 is coded by YSO classification, deduced as outlined in $\S 3$ above: green squares (class II), red squares (class I), blue squares (transition disk objects). Filled squares denote YSOs that are $\mathrm{H} \alpha$ emission-line objects. Of course, with $\mathrm{H} \alpha$ emission line measurements, the degeneracy between field stars and pre-main sequence class III cluster members is broken, so that class IIIs with $\mathrm{H} \alpha$ emission can be identified (blue $\times$ 's). The bright HAeBe stars $\mathrm{LkH} \alpha 324-\mathrm{SE}\left(\mathrm{V}=15.36, \mathrm{~A}_{V} \sim 10\right)$ and $\mathrm{LkH} \alpha 324\left(\mathrm{~V}=12.61, \mathrm{~A}_{V}=\right.$ $3.75)$ are identified by us as class I and class II, respectively. Even though the $K_{s}$-band error for $\mathrm{LkH} \alpha 324$ is slightly above our error threshold (error $=0.108$, threshold $=0.1$ ) we chose to include this object because of its prominence in the cluster. Most of the cluster associated with the emission-line stars lies in a region of low extinction to the east of the molecular cloud, or on the eastern edge of the cloud, where there is modest extinction, although there are bright, heavily extinguished members in the sample.

In Fig. 11, the $\mathrm{H} \alpha$ equivalent width $\mathrm{EW}[\mathrm{H} \alpha]$, a proxy for accretion strength (Herbig \& Dahm 2006), is plotted against the de-reddened SED slope from IRAC 3.6 to $8.0 \mu \mathrm{m}$ photometry for the 41 emission-line stars common to the list from Herbig \& Dahm (2006) and to our $\mathrm{NIR}+\mathrm{IRAC}$ dataset with uncertainties $\leq 0.1 \mathrm{mag}$ in all four IRAC bands. A vertical line at $\mathrm{EW}[\mathrm{H} \alpha]=10 \AA$ divides the plot into the single-value estimate of WTTS $(<10 \AA)$ and CTTS $(\geq 10 \AA)$. In their study of members of the young cluster IC 348, Lada et al. (2006) show that diskless stars (Class III) exhibit an IRAC determined SED slope of $<-2.56$, stars with optically thick disks exhibit slopes $>-1.8$, and they define sources with SED slopes between -1.8 and -2.56 to be 'anemic' disk objects (this class includes transition disk objects, optically thin disks etc.): we have adopted these parameters in Fig. 11, Generally, Class IIs (or Is) in L988e with optically thick disks show the strongest $\mathrm{H} \alpha$ equivalent widths, as they do in IC 348 (see also Hartmann (1998) who correlated $(K-L)$ excess emission from optically thick disks with large $\mathrm{EW}[\mathrm{H} \alpha]$ ). In order to compare our results with earlier studies, we have indicated by color our Class I, II, and III classifications. Although there is a general correlation of Class II/I with strong $\mathrm{H} \alpha$ equivalent widths in L988e, the figure also shows three stars with $\mathrm{H} \alpha$ equivalent widths $>10 \AA$, but slopes indicating class III according to the above definition, and three with $\mathrm{H} \alpha$ equivalent widths clearly $<10 \AA$, but slopes indicating thick disks (Class II). There are five objects in the anemic disk region. Since six objects do not obey the correlation described above, we now determine whether use of the single-valued $\mathrm{H} \alpha$ equivalent width definition of CTTS/WTTS is the cause of the departure. 
In the absence of spectroscopy for these 41 L988e cluster members, we made rough estimates of the emission line stars' spectral types from their measured $(V-I)$ colors (Herbig \& Dahm 2006) for objects where good $V$ and $I$ photometry was available. In order to do so, we first confirm that spectral types determined from $V$ and $I$ photometry can be roughly calibrated for stars with disks in young clusters.

The Lada et al. (2006) study of cluster members in IC 348 included published spectroscopic data, and they provide a table of median star-disk spectral energy distributions at wavelengths from $V$-band to $24 \mu \mathrm{m}$, all with a reddening of $\mathrm{A}_{V} \sim 2.5$, as a function of nine spectral type intervals. From their table, we confirm that the $(V-I)_{o}$ colors of both stars and objects with disks are sufficiently close for spectral typing into the nine intervals. We also examined the $J H K_{s}$ colors for the same median star-disk population in IC 348 (Lada et al. 2006), and found NIR colors to be unsatisfactory for rough spectral typing of YSOs, because, as expected, those colors can vary widely for photospheres, stars with 'anemic disks' and stars with thick disks.

Thirty-seven of the 41 emission line stars in L988e referred to above have good $V$ and $I$ photometry. We first de-redden the $V$ and $I$ magnitudes using our determined values of $\mathrm{A}_{K s}$, and assuming $\mathrm{A}_{V} / \mathrm{A}_{K s}=10$ (values of this ratio can vary by up to $30 \%$ (Glass 1999)). Although Herbig \& Dahm (2006) used a single value for $\mathrm{A}_{V}(2.5)$ with only a few exceptions, we found considerable variation in $\mathrm{A}_{K s}$ for these stars. Thus $(V-I)_{o}$ colors were estimated for these 37 emission-line stars, and $62 \%$ are later than $\mathrm{K} 7$ as determined from those colors and the rough spectral type calibration.

Applying the White \& Basri (2003) criterion for CTTS/WTTS classification as a function of rough spectral type, we found that 6 of the 37 objects yielded a different CTTS/WTTS classification than with the single-valued $\mathrm{H} \alpha$ equivalent width definition of CTTS/WTTS. These are indicated on Fig. 11] with the appropriate symbol encircled, and color-coded (green circle implies CTTS is the correct classification rather than WTTS, and a blue circle implies WTTS rather than CTTS). The resultant correlation of Class III with WTTS and Class II with CTTS is superior to that obtained using the single-valued $\mathrm{H} \alpha$ equivalent width discriminator. Three transition disk objects are identified and are indicated by a blue square. One of these is an anemic disk and one is a thick disk object. The remaining transition disk object is found slightly below the -2.56 slope delineation, rather than above, so is marginally non-compliant with the correlation discussed above. There are two additional objects which fall in the anemic disk region. Overall, the correlation of CTTS/WTTS to Class II/Class III is excellent once the revision to the equivalent width CTTS/WTTS definition proposed by White \& Basri (2003) is applied.

We now examine the statistics of the emission-line population. In addition to the 41 
objects discussed above, there are four $\mathrm{H} \alpha$ objects which do not have detections in all four IRAC bands and six which do have detections, but with uncertainties in IRAC bands $>0.1$ mag. There are also two emission-line objects in common with our IRAC $(3.6$ and $5.8 \mu \mathrm{m})$ + MIPS dataset in the vicinity of L988c. Of those objects with $\mathrm{H} \alpha$ emission, 28 are class IIs, two are transition disk objects, 19 are class IIIs, and two are class Is. Of the two in common with our IRAC $(3.6$ and $5.8 \mu \mathrm{m})+$ MIPS dataset, one is determined to be class I, and the other is determined to be class II from their $3-24 \mu \mathrm{m}$ SED slopes and ([5.8] - [24]) colors.

From these results, the disk fraction of just the emission-line population with NIR + IRAC counterparts is $67 \% \pm 20 \%$ including five which could be described as 'anemic disks' (Lada et al. 2006) based on IRAC SED slopes or MIPS $24 \mu \mathrm{m}$ detections. This percentage is consistent with either of the two age estimates cited by Herbig \& Dahm (2006), namely 0.6 and 1.7 Myr, using the curve of disk fraction to age from Hernandez et al. (2007).

\section{THE COOL DUST DISTRIBUTION}

Young cluster environments are permeated by dust associated with the natal molecular cloud, providing extinction as well as the cool dust emission detected at submillimeter wavelengths. All of the figures describing the molecular cloud will be used in the following discussion of the spatial distribution of the identified YSOs (Figs. 3, 4, and 5). The dust and molecular gas are distributed non-uniformly, and in order to characterize that distribution for comparison with the YSO distribution, we map the extinction $\left(\mathrm{A}_{K s}\right)$ in the neighborhood of L988e. The peak of the SCUBA $850 \mu \mathrm{m}$ emission (Fig. 4) coincides with the peak intensity of ${ }^{13} \mathrm{CO}$ emission, although a $\mathrm{NE}$ extension of the submillimeter emission does not have a parallel in the low spatial resolution molecular map: since the intensity of the emission varies on scales larger than the map size $\left(5^{\prime}\right)$ outside the peak, there is no information on the larger scale dust emission due to the iterative way the map is built, assuming that the edges chop into zero emission. Thus the SCUBA-measured emission is not ideal for illustrating the extent of the molecular cloud. Despite adequately mapping the extent of the cloud, available

low spatial resolution (50" beam size) CO emission line maps (Ridge et al. 2003) are also not ideal for comparing the dust distribution with the YSO distribution. In addition to inadequate spatial resolution, there are issues associated with low signal to noise in diffuse regions $\left(\mathrm{C}^{18} \mathrm{O}\right)$, and optical depth issues leading to saturation in dense cores $\left({ }^{13} \mathrm{CO}\right)$, as well as CO depletion due to chemistry and/or freeze-out in dense cores, and/or varying excitation temperature.

Although we could in principle utilize the IRAS $100 \mu \mathrm{m}$ HIRES map of the dust emission, it is more useful to use the higher spatial resolution $\left(H-K_{s}\right)$-derived extinction maps 
described in $\S 2$ to compare the extended dust distribution with the YSO distribution. Note that the map of $\mathrm{A}_{K s}$ (Fig. [5), at the same spatial resolution as the SCUBA map, shows the NE extension from the core indicating higher dust density, as seen by SCUBA. In addition, there are many small regions of enhanced extinction, and the extent of the molecular cloud defined by the $\mathrm{A}_{K s}$ map exceeds that suggested by the CO maps. All cloud tracers show an abrupt eastern edge to the cloud, which bifurcates the central cluster into the relatively high surface density population embedded within the cloud core, and the somewhat lower surface density population outside the cloud. This edge was likely produced by the two HAeBe stars: as stated by Herbig \& Dahm (2006), LkH $\alpha 324$ and 324SE 'have excavated a cavity on that edge and cleared out the area to the east, thereby exposing a small cluster of $\mathrm{H} \alpha$ emission-line stars.'

\section{SPATIAL DISTRIBUTION OF THE YOUNG STELLAR OBJECTS}

Classification of YSOs was executed as described in $\S 3$. We now examine the spatial distribution of the class Is and IIs so identified, first by overplotting their positions on a variety of images: namely, the IR images, the CO image, the cold dust emission images, and primarily, the extinction map.

Although the overall stellar density in the composite NIR image (Fig. 2) is substantially lower toward the molecular cloud, the peak surface density of YSOs is found toward the molecular cloud core, as identified by all of the molecular cloud tracers. LkH $\alpha 324$ and LkH $\alpha 324 \mathrm{SE}$ are associated with that high density region, which we call the 'embedded' population of the cluster.

To illustrate this more clearly, YSO surface density distributions are formed for all YSOs, utilizing the method developed for NGC 7129, GGD12-15, and IRAS20050+2720, three clusters previously studied in the Young Cluster Project (Gutermuth et al. 2005). Because there exists a wide range in stellar surface densities over a young cluster, a method which utilizes adaptive smoothing lengths allows both the highest and lowest stellar density regions to be probed. At each sample position $[i, j]$ in a uniform grid the projected radial distance to the $N$ th nearest star, $r_{N}(i, j)$ is measured, and the local surface density $\sigma(i, j)=$ $N /\left(\pi r_{N}^{2}(i, j)\right)$ is calculated. Here the grid size is $3^{\prime \prime}$, and $N=5$. A value of $N \geq 5$ is chosen to retain sufficient spatial resolution. On the other hand (Gutermuth 2005), $N$ must be as small as feasible to allow small, high density sub-groups to be properly represented. Also

see Gutermuth et al. (2004). The grid size selection of $3^{\prime \prime}$ was chosen to fully resolve all structure at the smallest scales (i.e. highest densities) in the $N=5$ density distribution. The surface density of YSOs is compared with a SCUBA gray-scale image in Fig. 12 of the 
cool dust ridge associated with the edge of the molecular cloud. The surface density contours reveal an asymmetric cluster, with the highest surface density region (peaking at $\sim 1706$ stars $\mathrm{pc}^{-2}$ ), including mostly class IIs, concentrated at the peak of the dust emission. $\mathrm{LkH} \alpha$ 324 is located on the northern edge of this region and $\mathrm{LkH} \alpha 324 \mathrm{SE}$ lies on the eastern edge. A second, less compact surface density region associated with the emission-line population (peaking at $\sim 747 \mathrm{stars}^{-2}$ ) is found to the east of the dust ridge: these are also primarily class II objects (note that class III objects are not included in this figure, whereas in the

emission-line selected population (Herbig \& Dahm 2006) overplotted on the map of $\mathrm{A}_{K s}$ in Fig. 5, both class II and III stars are included). This population falls in the cavity where the molecular cloud has been excavated, presumably by the bright HAeBe stars LkH $\alpha 324$ and $324 \mathrm{SE}$. We call this the 'exposed' cluster population.

The exposed population of the asymmetric cluster may be somewhat older than the embedded population, as suggested by the relatively lower YSO stellar densities encountered, as well as the necessity that enough time has passed to sculpt the cloud in the vicinity of the exposed cluster. We examine this further in the next section by estimating disk fractions for the exposed and embedded populations in a circular region surrounding the surface density peaks, using standard methods.

In addition to these concentrated distributions of YSOs, in both the excavated cavity, and in the dense molecular knot, a distributed halo of YSOs is found mainly in the diffuse portions of the associated molecular cloud, and another to the east of the cloud. These haloes include predominantly class IIs, but the presence of class Is in the diffuse molecular cloud is important to note. A much larger study focusing on the distributed population of YSOs well beyond the clusters, particularly the class Is, is underway. There we hope to determine whether the class Is in the distributed population formed in situ, or were ejected from the clusters.

\section{DISK FRACTIONS IN THE EXPOSED AND EMBEDDED CLUSTER REGIONS}

We show in this section that $\sim 55 \pm 16 \%$ of the objects in a $1.5^{\prime}$ diameter circle centered at $\left[21^{h} 4^{m} 4.5^{s}, 50^{\circ} 14^{\prime} 32^{\prime \prime} .7\right]$ are class IIs: this region corresponds to the peak of the exposed population of the cluster (here we have purposely not included the emission-line objects associated with substantial extinction at the edge of the L988e dark cloud, or the extended diffuse halo population, in contrast to the treatment in $\S 4$ ). This disk fraction, while estimated more rigorously than in $\S 4$, entails only a relatively small number of stars because L988e is sparsely populated. 
In contrast, the disk fraction in a similar sized region centered on the embedded population's peak density (centered at $\left[21^{h} 3^{m} 52.97^{s}, 50^{\circ} 14^{\prime} 43^{\prime \prime} .5\right]$ ) is $\sim 74 \pm 15 \%$. At face value, the higher mean disk fraction indicates the embedded population is younger than the exposed population, however, because of small number statistics, the large uncertainties overlap, so we cannot be certain of the relative ages of the regions.

Disk fractions were estimated in the following manner. $K_{s}$-band magnitude histograms $(\mathrm{KMH})$ were created for $1.5^{\prime}$ diameter regions around the peaks of the exposed and embedded populations and for three $3^{\prime}$ diameter regions off cloud that do not contain any known protostars, stars with disks, or $\mathrm{H} \alpha$ emission objects (see Fig. 2 for the locations of these regions and Fig. 13 for the histograms). To determine the magnitude at which field star contamination, begins to dominate the cluster $\mathrm{KMH}$, we consider the faintest magnitude bin in the cluster $\mathrm{KMH}$ greater than $3 \sigma_{\text {background, }}$ assuming Poisson counting statistics for each magnitude bin in the KMHs. This limit occurs at $14^{\text {th }}$ magnitude. The average $K_{s}$-band extinction for the exposed population, the off-cloud regions, and the embedded population

are $\mathrm{A}_{K s}=0.37, \mathrm{~A}_{K s}=0.28$, and $\mathrm{A}_{K s}=1.3$, respectively, estimated from $J, H, K_{s}$, colors as above (§3). To account for the higher extinction toward the embedded population, the offcloud sources are artificially extinguished to the mean extinction of the embedded population before subtracting the off-cloud KMH from the embedded population KMH. By subtracting the off-cloud KMH from the exposed population KMH for the magnitude bins less than the $14^{\text {th }}$ magnitude limit, we estimate 22 cluster members in the exposed population, and 19 cluster members in the embedded population: these values are used in the disk fraction determination above.

\section{COMPARISON WITH OTHER CLUSTERS}

We restrict comparison of L988e to six young clusters (Gutermuth 2005) which have been studied in many of the same ways as L988e, although L988e is the only cluster with exposed stars which have been studied for $\mathrm{H} \alpha$ emission. Of those six, two filamentary clusters, GGD12-15 and IRAS 20050+2720, have the following properties: the class Is follow closely the asymmetric distribution defined by the dust ridges in their respective molecular clouds, while the class IIs are more dispersed along the filament. The two clusters widely vary in far-IR luminosity and cloud mass, and range from moderate to relatively high spatial density ( $\sim 1200$ and $3000 \mathrm{pc}^{-2}$ peak respectively). GGD 12-15 has 102 YSOs, of which $25 \%$ are class I, while IRAS $20050+2720$ has 121 YSO members, of which $21 \%$ are class I: class I number counts may be biased toward lower numbers due to the lack of MIPS coverage when these regions were analyzed. Two other clusters, NGC 7129 and AFGL 490, 
are more diffuse than the filamentary clusters, with little high density clustering and little asymmetric structure. There are a total of 63 and 98 YSOs respectively in NGC 7129 and AFGL 490, of which $17 \%$ and $13 \%$ are class I respectively, with the same bias toward lower class I number counts due to lack of MIPS coverage. YSO spatial distribution in the L988e exposed cluster is similar to that in the latter two clusters, especially to that of NGC 7129: in particular, a large population of class IIs clustered in a cavity in the molecular cloud near a Be star LkH $\alpha 234$, resembles the exposed population discussed here. However, L988e does exhibit some asymmetry, and there is a substantial number of class Is in the L988e embedded cluster population. The distribution of class Is resembles those in GGD12-15 and IRAS 20050+2720: class Is are found in both the high density dusty region near $\mathrm{LkH} \alpha$ 324 and 324-SE, and throughout the rest of the molecular cloud. However, in contrast to GGD12-15 and IRAS 20050+2720, only $10 \%$ of the 79 class I and II objects identified by IRAC and MIPS in all of L988e are class I. Of these four clusters, only IRAS $20050+2720$ has comparable FIR luminosity to L988e: the other three are $\sim 6$ - 30 times more luminous. NGC 7129 is estimated to be $\sim 3$ Myr in age, while the filamentary clusters are thought to be younger. The final two young clusters studied in this way, CepA and MonR2, have an order

of magnitude higher FIR luminosity, but are otherwise different from each other. MonR2 exhibits a filamentary distribution of 49 class Is (20\% of the total) similar to that observed for GGD12-15 and IRAS 20050+2720, whereas CepA is sparsely populated by 8 class Is (only $9 \%$ of total YSOs) while the 77 class IIs are anti-associated with CO density enhancements. A comprehensive spatial distribution study of the entire Young Cluster Survey is in preparation.

\section{SUMMARY}

1. Spitzer photometry of the asymmetric L988e cluster in combination with new NIR photometry reveals the presence of an embedded population associated with the molecular cloud (the cloud is defined by $\mathrm{CO}$ emission, cool dust emission, and $\mathrm{A}_{K s}$ maps of extinction). This is in addition to the previously studied exposed region of the cluster, associated with the optically studied $\mathrm{H} \alpha$ emission-line stars in a cloud cavity on the eastern edge, sculpted presumably by the HAeBe stars $\mathrm{LkH} \alpha 324$ and $\mathrm{LkH} \alpha 324 \mathrm{SE}$.

2. The disk fraction (class II/(class II + class III) of ONLY the emission-line objects, primarily located in the exposed region, is $\sim 67 \%$. This is consistent with either of the two age estimates deduced from $V$ and $I$ photometry (Herbig \& Dahm 2006).

3. The embedded population exhibits larger surface density than the exposed population of the cluster, and is fully enshrouded within L988e's natal molecular cloud. This, as well as the higher $\underline{\text { mean }}$ disk fraction calculated in the conventional way for all cluster members 
in the field ( $74 \%$ vs. $55 \%$ ) points to the probability of a somewhat younger age for the embedded population as compared with the exposed population, although the uncertainties of these disk fractions overlap. The exposed population of the cluster lies primarily in a region where the extinction $\mathrm{A}_{K s}<0.5$ mag. On the other hand, the embedded population suffers a peak extinction of $\mathrm{A}_{K s}=3$, and extinctions $\mathrm{A}_{K s}>0.5$ throughout the molecular cloud.

4. In addition to the asymmetric cluster, there exists a diffuse halo population of YSOs surrounding both the exposed and embedded regions of the cluster. Although the halo is dominated by class IIs, class Is are also found in the diffuse halo. Whether these halo objects are formed in situ is yet to be determined.

5. Comparing these populations to six regions in the Young Cluster Program studied in a similar manner (Gutermuth 2005), we confirm the conclusion that the spatial distribution within the cluster is not strongly coupled to either the FIR luminosity or the numbers of cluster members. The younger clusters more faithfully follow the cloud density structure in which the cluster is born, and exhibit higher surface density. Therefore, L988e is older than the youngest clusters in the survey because the Eastern part of the cloud has had time to be excavated. It is becoming increasingly obvious that class Is are found in the haloes around clusters in regions where the molecular column density is relatively low.

6. Spitzer/IRAC $3.6 \mu \mathrm{m}, 5.8 \mu \mathrm{m}$ and Spitzer/MIPS $24 \mu \mathrm{m}$ photometry reveal another embedded cluster associated with the object L988c.

This research has made use of the NASA/IPAC Infrared Science Archive, which is operated by the Jet Propulsion Laboratory, California Institute of Technology, under contract with the National Aeronautics and Space Administration. This publication makes use of data products from the Two Micron All Sky Survey, which is a joint project of the University of Massachusetts and the Infrared Processing and Analysis Center/California Institute of Technology, funded by the National Aeronautics and Space Administration and the National Science Foundation. JPW acknowledges support from NSF grant AST-0324323, and JLP support from SAO/JPL SV4-74011.

\section{REFERENCES}

Allen, L. E., Calvet, N., D’Alessio, P., Merin, B., Hartmann, L., Megeath, S. T., Gutermuth, R. A., Muzerolle, J., Pipher, J. L., Myers, P. C.; Fazio, G. G. 2004, ApJS, 154, 363

Baraffe, I., et al., 1998, å, 337, 403 
Clark, F. O., 1986, A\&A, 164, L19

Carpenter, J. M., 2001, AJ, 121, 2851

Dobashi, K., Bernard, J., Yonekura, Y.Fukui, Y., 2004, ApJS, 95, 419

D'Antona, F. \& Mazzitelli, I., 1997, Cool Stars in Clusters and Associations, ed. G. Micela \& R. Pallavicini (Firenzw: Soc. Astr. Italiana), 807

Elston, R., J., et al., 2006, ApJ, 639, 816

Fazio, G., G., et al., 2004, ApJ, 154, 39

Flaherty, K. et al., 2007, ApJ. in press

Glass, I., Handbook of Infrared Astronomy (Cambridge: Cambridge Univ. Press)

Gutermuth, R. A., Megeath, S. T.,Muzerolle, J., Allen, L. E., Pipher, J. L., Myers, P. C., Fazio, G. G., 2004, ApJS, 154, 374

Gutermuth, R. A., 2005, PhD, University of Rochester

Gutermuth, R.A., Megeath, S.T., Pipher, J.L., Williams, J.P., Allen, L.E., Myers, P.C.\& Raines, S.N.., 2005, ApJ, 632, 397

Gutermuth, R.A. et al., 2007, in preparation.

Hartmann, L., 1998, Accretion Processes in Star Formation, (Cambridge University Press: Cambridge), p. 124.

Herbig, G. H.\& Bell, K. R., 1988, Lick Obs. Bull, n.1111, 1

Herbig, G.H. \& Daum, S.E., 2006, AJ, 131, 1530.

Hernandez, J., Hartmann, L., Megeath, T., Gutermuth, R., Muzerolle, J., Calvet, N., Vivas, A.K., Briceno, C., Allen, L., Stauffer, J., Young, E., and Fazio, G., submitted to ApJ.

Hodapp, K., 1994, ApJS, 94, 615

Indebetouw, R., Mathis, J. S., Babler, B.L., Meade, M. R., Watson, C., Whitney, B. A., Wolff, M. J., Wolfire, M. G., Cohen,M., Bania, T. M., and 10 coauthors, 2005, ApJ, 619,9311

Jenness, T., Lightfoot, J. F., Holland, W. S.1998, SPIE 3357, 548 
Lada, C. J., \& Lada E. A., 2003, ARA\&A, 41, 57

Lada, C. J., et al., 2006, AJ, 131, 1574

Meyer, M. R., Calvet, N., \& Hillenbrand, L. A., 1997, AJ, 114, 288

Muzerolle, J., Megeath, S. T.,Gutermuth, R. A., Allen, L. E., Pipher, J. L., Hartmann, L., Gordon, K. D., Padgett,D. L., Noriega-Crespo, A., Myers, P. C., Fazio, G. G., Rieke, G. H., Young, E. T.,Morrison, J. E., Hines, D. C., Su, K. Y. L., Engelbracht, C. W., Misselt, K. A.,2004, ApJS, 154, 379

Pipher, J. L., et al., 2004, SPIE 5487, 234

Porras, A., Christopher, M., Allen, L., DiFrancesco, J., Megeath, S. T., Myers, P. C., 2003, AJ, 126,1916

Reach, W.T., Megeath, S.T., Cohen, M., Hora, J., Carey, S., Surace, J., Willner, S.P., Barmby, P., Wilson, G., Glaccum, W., Lowrance, P., Marengo, M., and Fazio, G.G., 2005, PASP, 117, 978.

Ridge, N. A.. Wilson, T. L., Megeath, S. T.,Allen, L. E., Myers, P. C., 2003, AJ, 126, 286.

Siess, L., Dufour, E., \& Forestini, M. 2000, A\&A, 358, 593

Spitzer Science Center. 2005, Multiband Imaging Photometer for Spitzer (MIPS) Data Handbook (Pasadena: SSC), http://ssc.spitzer.caltech.edu/mips/dh/

White, R. J. \& Basri, G. 2003, ApJ, 582, 1109

Whitney, B. A., Wood, K., Bjorkman, J. E.,Cohen, M., 2003, ApJ, 598, 1079

Whitney, B. A., et al., 2004, ApJS, 154, 315

Wilson, J. C., Eikenberry, S. S., Henderson,C. P., Hayward, T. L., Carson, J. C.; Pirger, B. Barry, D. J., Brandl, B. R.,Houck, J. R., Fitzgerald, G. J., Stolberg, T. M., 2003, SPIE, 4841, 451 
Table 1. L988e Cluster Characteristics Compiled from the Literature.

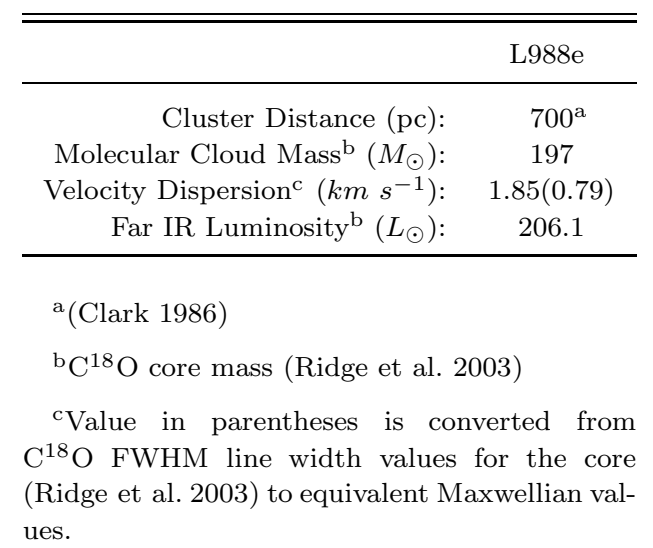


Table 2. Objects with Mid Infrared Excesses (extragalactic contaminants removed): Class I

\begin{tabular}{|c|c|c|c|c|c|c|c|c|c|c|c|c|}
\hline $\mathrm{DEC}_{2000}$ & $J$ & $H$ & $K$ & {$[3.6]$} & {$[4.5]$} & {$[5.8]$} & {$[8.0]$} & {$[24]$} & $\mathrm{A}_{K s}^{\mathrm{b}}$ & $\alpha_{I R A C}{ }^{\mathrm{c}}$ & $\mathrm{IH} \alpha^{\mathrm{d}}$ & $\operatorname{EW}(\mathrm{H} \alpha)^{\mathrm{e}}$ \\
\hline 21:02:50.50 $\pm 0.2350: 18: 52.43 \pm 0.06$ & $\cdots$ & $\cdots$ & $\cdots$ & $10.50 \pm 0.003$ & $\cdots$ & $9.66 \pm 0.004$ & $\cdots$ & $5.44 \pm 0.011$ & $\cdots$ & $-0.41^{g}$ & $\cdots$ & $\cdots$ \\
\hline $21: 02: 52.00 \pm 0.32 \quad 50: 13: 43.96 \pm 0.19$ & $\cdots$ & $\cdots$ & $\cdots$ & $10.59 \pm 0.004$ & $\cdots$ & $9.48 \pm 0.004$ & $\cdots$ & $5.59 \pm 0.014$ & $\cdots$ & $-0.48^{\mathrm{g}}$ & 623 & 26. \\
\hline 21:02:52.28 $\pm 0.0550: 12: 29.47 \pm 0.12$ & $\cdots$ & $\cdots$ & $\cdots$ & $8.55 \pm 0.002$ & $\cdots$ & $7.24 \pm 0.002$ & $\cdots$ & $2.55 \pm 0.003$ & $\cdots$ & $0.020^{\mathrm{g}}$ & $\cdots$ & $\cdots$ \\
\hline 21:02:54.17 $\pm 0.25 \quad 50: 12: 27.46 \pm 0.10$ & $\cdots$ & $\cdots$ & $\cdots$ & $12.49 \pm 0.007$ & $\cdots$ & $10.01 \pm 0.005$ & $\cdots$ & $4.36 \pm 0.008$ & $\cdots$ & $0.960^{\mathrm{g}}$ & $\cdots$ & $\cdots$ \\
\hline 21:02:56.60土0.14 50:11:59.22 \pm 0.04 & $\cdots$ & $\cdots$ & $\cdots$ & $10.55 \pm 0.005$ & $\cdots$ & $9.65 \pm 0.007$ & $\cdots$ & $5.40 \pm 0.014$ & $\cdots$ & $-0.37^{\mathrm{g}}$ & $\cdots$ & $\cdots$ \\
\hline 21:02:58.06 $\pm 0.1950: 15: 25.63 \pm 0.17$ & $\cdots$ & $\cdots$ & $\cdots$ & $9.91 \pm 0.002$ & $\cdots$ & $7.82 \pm 0.002$ & $\cdots$ & $3.57 \pm 0.004$ & $\cdots$ & $0.080^{\mathrm{g}}$ & $\cdots$ & $\cdots$ \\
\hline 21:03:00.19 \pm 0.25 50:21:56.59 \pm 0.13 & $\cdots$ & $\cdots$ & $\cdots$ & $11.80 \pm 0.004$ & $\cdots$ & $9.56 \pm 0.004$ & $\cdots$ & $4.03 \pm 0.006$ & $\cdots$ & $0.800^{\mathrm{g}}$ & $\cdots$ & $\cdots$ \\
\hline 21:03:01.16 $\pm 0.0150: 12: 29.25 \pm 0.03$ & $\cdots$ & $\cdots$ & $\cdots$ & $11.60 \pm 0.004$ & $\cdots$ & $10.19 \pm 0.005$ & $\cdots$ & $5.67 \pm 0.018$ & $\cdots$ & $-0.04^{\mathrm{g}}$ & $\cdots$ & $\cdots$ \\
\hline 21:03:02.98 $\pm 0.15 \quad 50: 12: 16.36 \pm 0.18$ & $\cdots$ & $\cdots$ & $\cdots$ & $12.60 \pm 0.007$ & $\cdots$ & $11.59 \pm 0.014$ & $\cdots$ & $7.62 \pm 0.076$ & $\cdots$ & $-0.47^{\mathrm{g}}$ & $\cdots$ & $\cdots$ \\
\hline 21:03:03.24 $\pm 0.1250: 13: 12.66 \pm 0.06$ & $\cdots$ & $\cdots$ & $\cdots$ & $9.79 \pm 0.004$ & $\cdots$ & $8.60 \pm 0.041$ & $\cdots$ & $2.95 \pm 0.014$ & $\cdots$ & $0.470^{\mathrm{g}}$ & $\cdots$ & $\cdots$ \\
\hline 21:03:03.34 \pm 0.43 50:09:31.94 \pm 0.15 & $\cdots$ & $\cdots$ & $\cdots$ & $12.92 \pm 0.007$ & $712.26 \pm 0.006$ & $611.61 \pm 0.012$ & $10.48 \pm 0.011$ & & $\cdots$ & -0.05 & $\cdots$ & $\cdots$ \\
\hline 21:03:06.85 $\pm 0.1750: 12: 05.20 \pm 0.37$ & $\cdots$ & $\cdots$ & $\cdots$ & $11.77 \pm 0.005$ & $511.29 \pm 0.004$ & $410.60 \pm 0.008$ & $9.54 \pm 0.005$ & $6.72 \pm 0.022$ & $\cdots$ & -0.25 & $\cdots$ & $\cdots$ \\
\hline 21:03:13.90 $\pm 0.2150: 12: 46.78 \pm 0.06$ & $\cdots$ & $\cdots$ & $\cdots$ & $11.37 \pm 0.004$ & $410.57 \pm 0.004$ & $410.03 \pm 0.006$ & $9.20 \pm 0.005$ & $6.16 \pm 0.015$ & $\ldots$ & -0.40 & $\cdots$ & $\cdots$ \\
\hline 21:03:22.92 $\pm 0.22 \quad 50: 11: 31.25 \pm 0.23$ & $\cdots$ & $\cdots$ & $\cdots$ & $16.33 \pm 0.066$ & $15.19 \pm 0.052$ & $214.06 \pm 0.063$ & $12.96 \pm 0.067$ & $9.60 \pm 0.151$ & $\cdots$ & 1.040 & $\cdots$ & $\cdots$ \\
\hline 21:03:35.66 \pm 0.24 50:15:25.77 \pm 0.33 & $\cdots$ & $16.57 \pm 0.066$ & $6615.59 \pm 0.039$ & $13.92 \pm 0.030$ & $13.28 \pm 0.017$ & $712.92 \pm 0.096$ & $11.93 \pm 0.049$ & $6.52 \pm 0.026$ & 1.09 & -0.64 & $\ldots$ & $\cdots$ \\
\hline 21:03:53.44 $\pm 0.42 \quad 50: 14: 47.55 \pm 0.39$ & $\cdots$ & $\ldots$ & $\ldots$ & $\ldots$ & $8.68 \pm 0.008$ & $7.00 \pm 0.018$ & $5.81 \pm 0.028$ & $1.35 \pm 0.042$ & $\ldots$ & $0.98^{\mathrm{f}}$ & $\cdots$ & $\ldots \stackrel{N}{\Perp}$ \\
\hline 21:03:56.37 $\pm 0.02 \quad 50: 16: 03.27 \pm 0.08$ & $\cdots$ & $\cdots$ & $\cdots$ & $13.59 \pm 0.013$ & $11.25 \pm 0.005$ & $59.90 \pm 0.007$ & $8.62 \pm 0.007$ & $3.97 \pm 0.024$ & $\cdots$ & 2.720 & $\cdots$ & $\cdots \quad \mid$ \\
\hline 21:03:58.13 $\pm 0.3450: 14: 39.77 \pm 0.50$ & $7.71 \pm 0.015$ & $6.51 \pm 0.003$ & $3 \quad 5.32 \pm 0.011$ & $4.42 \pm 0.006$ & $3.64 \pm 0.005$ & $1.96 \pm 0.001$ & $1.65 \pm 0.001$ & $\cdots$ & 1.14 & 0.580 & 651 & 159 \\
\hline 21:04:00.97 $\pm 0.4850: 15: 56.77 \pm 0.08$ & $14.09 \pm 0.005$ & $513.08 \pm 0.00$ & $0212.23 \pm 0.001$ & $10.25 \pm 0.002$ & $9.50 \pm 0.003$ & $8.79 \pm 0.009$ & $7.79 \pm 0.005$ & $4.13 \pm 0.027$ & 0.68 & -0.03 & 657 & 69. \\
\hline
\end{tabular}

${ }^{a}$ Magnitudes are de-reddened when extinction value $\mathrm{A}_{K s}$ quoted.

${ }^{\mathrm{b}} \mathrm{A}_{K}$ determined from method outlined in $\S 5$.

cSlope $\alpha$ determined from IRAC photometry

${ }^{\mathrm{d}}$ Running number from Herbig \& Dahm (2006).

${ }^{\mathrm{e}} \mathrm{EW}[\mathrm{H} \alpha]$ from Herbig \& Dahm (2006).

${ }^{\mathrm{g}}$ Slope $\alpha$ determined from [3.6], [5.8] and [24] photometry

${ }^{\mathrm{f}}$ Slope $\alpha$ determined from [4.5] to [24] photometry 
Table 3. Objects with Mid Infrared Excesses (extragalactic contaminants removed): Class II

\begin{tabular}{|c|c|c|c|c|c|c|c|c|c|c|c|c|}
\hline $\mathrm{DEC}_{2000}$ & $J$ & $H$ & $K$ & {$[3.6]$} & {$[4.5]$} & {$[5.8]$} & {$[8.0]$} & {$[24]$} & $\mathrm{A}_{K s} \mathrm{~b}$ & $\alpha_{I I}$ & $\mathrm{IH} \alpha^{\mathrm{d}}$ & $\mathrm{EW}(\mathrm{H} \alpha)^{\mathrm{e}}$ \\
\hline $21: 02: 55.21 \pm 0.05 \quad 50: 11: 55.22 \pm 0.22$ & $\ldots$ & $\ldots$ & $\ldots$ & $9.06 \pm 0.006$ & & $7.68 \pm 0.004$ & & $4.34 \pm 0.008$ & & $-0.66^{\mathrm{f}}$ & 624 & 29. \\
\hline $21: 02: 59.28 \pm 0.03 \quad 50: 10: 30.24 \pm 0.13$ & $\ldots$ & $\ldots$ & $\ldots$ & $11.61 \pm 0.004$ & & $10.70 \pm 0.007$ & $\ldots$ & $6.94 \pm 0.061$ & & $-0.62^{\mathrm{f}}$ & $\ldots$ & $\ldots$ \\
\hline 21:03:06.34 $\pm 0.24 \quad 50: 10: 04.04 \pm 0.08$ & $\cdots$ & $\cdots$ & $\cdots$ & $11.06 \pm 0.004$ & $410.48 \pm 0.004$ & $4 \quad 9.99 \pm 0.005$ & $9.59 \pm 0.005$ & $7.87 \pm 0.047$ & . . & -1.16 & $\ldots$ & $\ldots$ \\
\hline 21:03:08.09 \pm 0.15 50:10:01.71 \pm 0.20 & $\cdots$ & $\cdots$ & $\cdots$ & $14.31 \pm 0.020$ & $13.92 \pm 0.029$ & $13.57 \pm 0.062$ & $13.39 \pm 0.093$ & $\cdots$ & $\ldots$ & -1.78 & $\cdots$ & $\cdots$ \\
\hline $21: 03: 20.22 \pm 0.4950: 15: 58.91 \pm 0.43$ & $9.94 \pm 0.008$ & $9.34 \pm 0.001$ & $9.22 \pm 0.001$ & $8.49 \pm 0.002$ & $8.14 \pm 0.003$ & $7.60 \pm 0.003$ & $6.64 \pm 0.002$ & $3.55 \pm 0.005$ & 2.15 & -0.70 & $\ldots$ & $\ldots$ \\
\hline 21:03:21.78 \pm 0.27 50:09:41.73 \pm 0.10 & $15.06 \pm 0.011$ & $14.36 \pm 0.005$ & $14.05 \pm 0.007$ & $13.38 \pm 0.013$ & $313.18 \pm 0.029$ & $913.16 \pm 0.053$ & $13.17 \pm 0.070$ & $\cdots$ & 0.53 & -2.64 & $\cdots$ & $\cdots$ \\
\hline 21:03:23.72 $\pm 0.17 \quad 50: 17: 21.65 \pm 0.56$ & $10.24 \pm 0.088$ & $89.60 \pm 0.004$ & $9.40 \pm 0.001$ & $9.09 \pm 0.003$ & $8.73 \pm 0.003$ & $8.29 \pm 0.005$ & $7.72 \pm 0.004$ & $5.94 \pm 0.021$ & 3.56 & -1.25 & $\cdots$ & $\cdots$ \\
\hline 21:03:24.17 $\pm 0.32 \quad 50: 11: 01.01 \pm 0.34$ & $\cdots$ & & $\cdots$ & $12.81 \pm 0.006$ & $612.11 \pm 0.005$ & $511.65 \pm 0.015$ & $11.77 \pm 0.061$ & $\cdots$ & $\cdots$ & -1.65 & $\cdots$ & $\ldots$ \\
\hline 21:03:26.47 $\pm 0.37 \quad 50: 14: 28.25 \pm 0.60$ & $15.06 \pm 0.012$ & $214.22 \pm 0.005$ & $13.68 \pm 0$ & $12.79 \pm 0.008$ & $812.44 \pm 0.008$ & $812.03 \pm 0.020$ & $11.52 \pm 0.026$ & $7.76 \pm 0.043$ & 0.87 & -1.37 & $\cdots$ & $\cdots$ \\
\hline 21:03:28.67 \pm 0.78 50:12:34.94 \pm 0.47 & $14.26 \pm 0.064$ & $413.36 \pm 0.009$ & $12.70 \pm 0.005$ & $12.31 \pm 0.017$ & $712.16 \pm 0.015$ & $512.06 \pm 0.037$ & $12.13 \pm 0.088$ & $\cdots$ & 1.81 & -2.64 & $\ldots$ & $\cdots$ \\
\hline 21:03:30.74 $\pm 0.70 \quad 50: 12: 49.04 \pm 0.54$ & $12.52 \pm 0.046$ & $611.80 \pm 0.004$ & $11.46 \pm 0$ & $11.00 \pm 0$ & $510.73 \pm 0$ & $510.22 \pm 0.010$ & $9.66 \pm 0.008$ & $7.45 \pm 0.051$ & 2.31 & -1.26 & $\ldots$ & $\ldots$ \\
\hline 21:03:36.20 \pm 0.48 50:15:56.67 \pm 0.33 & $13.24 \pm 0.005$ & $512.50 \pm 0$ & $12.12 \pm 0$ & 11.3 & $410.98 \pm 0$ & $410.51 \pm 0.009$ & $9.92 \pm 0.010$ & $7.83 \pm 0.072$ & 1.07 & -1.14 & $\cdots$ & $\cdots$ \\
\hline 21:03:36.24 $\pm 0.81 \quad 50: 11: 50.14 \pm 0.53$ & $16.57 \pm 0.035$ & $15.72 \pm 0$ & $15.15 \pm 0$ & 14.2 & $9 \pm 0$ & 513.41 & $12.56 \pm 0.064$ & $\cdots$ & 0.47 & -0.90 & $\ldots$ & $\ldots$ \\
\hline $21: 03: 36.69 \pm 0.45 \quad 50: 16: 49.22 \pm 0.13$ & $14.58 \pm 0.035$ & $513.76 \pm 0.007$ & $13.23 \pm 0$ & $12.36 \pm 0.007$ & $712.12 \pm 0.007$ & $711.65 \pm 0.018$ & $11.12 \pm 0.022$ & $8.30 \pm 0.080$ & 1.40 & -1.38 & $\ldots$ & $\ldots$ \\
\hline $21: 03: 37.44 \pm 0.56 \quad 50: 13: 36.68 \pm 0.37$ & $\ldots$ & $13.99 \pm 0.028$ & $13.69 \pm 0.015$ & $13.24 \pm 0.017$ & $713.12 \pm 0.015$ & $512.73 \pm 0.066$ & $12.56 \pm 0.081$ & $\ldots$ & 2.31 & -2.01 & $\ldots$ & $\ldots$ \\
\hline $21: 03: 39.47 \pm 0.32 \quad 50: 15: 52.84 \pm 0.27$ & $12.40 \pm 0.001$ & $11.67 \pm 0.019$ & $11.30 \pm 0.022$ & $10.69 \pm 0.002$ & $210.52 \pm 0.003$ & $310.07 \pm 0.006$ & $9.48 \pm 0.008$ & $6.78 \pm 0.059$ & 0.14 & -1.41 & 631 & 55. ज \\
\hline $21: 03: 39.68 \pm 0.21 \quad 50: 12: 36.28 \pm 0.55$ & & $12.40 \pm 0.028$ & $11.98 \pm 0.009$ & $11.50 \pm 0.008$ & $811.28 \pm 0.009$ & $910.78 \pm 0.024$ & $10.43 \pm 0.054$ & & 3.26 & -1.55 & $\ldots$ & $\cdots \quad \mid$ \\
\hline $21: 03: 40.70 \pm 0.1150: 13: 54.05 \pm 0.05$ & $11.19 \pm 0.001$ & $10.54 \pm 0.001$ & $10.31 \pm 0.022$ & $9.73 \pm 0.003$ & $9.48 \pm 0.003$ & $9.09 \pm 0.004$ & $8.57 \pm 0.004$ & $6.03 \pm 0.026$ & 1.18 & -1.49 & $\ldots$ & $\ldots$ \\
\hline $21: 03: 43.58 \pm 0.27 \quad 50: 15: 58.23 \pm 0.00$ & $11.01 \pm 0.001$ & $10.16 \pm 0.017$ & $9.60 \pm 0.019$ & $8.25 \pm 0.002$ & $7.82 \pm 0.002$ & $7.35 \pm 0.002$ & $6.97 \pm 0.002$ & $4.25 \pm 0.012$ & 0.77 & -1.36 & 632 & 39. \\
\hline $21: 03: 44.23 \pm 0.18 \quad 50: 16: 05.00 \pm 0.53$ & $14.52 \pm 0.030$ & $13.92 \pm 0.008$ & $13.92 \pm 0.010$ & $13.17 \pm 0.039$ & $12.98 \pm 0.066$ & $\cdots$ & $\ldots$ & ... & 1.46 & .. & $\ldots$ & $\ldots$ \\
\hline $21: 03: 44.46 \pm 0.3450: 15: 51.63 \pm 0.33$ & $12.28 \pm 0.002$ & $211.60 \pm 0.001$ & $11.31 \pm 0.001$ & $10.66 \pm 0.009$ & $10.23 \pm 0.007$ & $7 \quad 9.81 \pm 0.010$ & $9.22 \pm 0.009$ & $\cdots$ & 0.99 & -1.19 & 633 & 156 \\
\hline $21: 03: 46.39 \pm 0.24 \quad 50: 13: 44.79 \pm 0.05$ & $15.61 \pm 0.003$ & $314.77 \pm 0.002$ & $14.22 \pm 0.002$ & $13.50 \pm 0.015$ & $13.15 \pm 0.018$ & $812.81 \pm 0.050$ & $12.35 \pm 0.084$ & $7.85 \pm 0.155$ & 0.09 & -1.52 & & $\ldots$ \\
\hline $21: 03: 46.58 \pm 0.27 \quad 50: 08: 41.97 \pm 0.82$ & $12.51 \pm 0.001$ & $111.85 \pm 0.001$ & $11.60 \pm 0.001$ & $11.32 \pm 0.004$ & $11.15 \pm 0.004$ & $410.87 \pm 0.012$ & $10.25 \pm 0.036$ & $7.57 \pm 0.115$ & 0.15 & -1.62 & 635 & 4.2 \\
\hline 21:03:46.71 $\pm 0.43 \quad 50: 15: 26.33 \pm 0.24$ & $\cdots$ & $13.22 \pm 0.009$ & $12.33 \pm 0.004$ & $11.01 \pm 0.005$ & $10.43 \pm 0.004$ & $49.72 \pm 0.014$ & $8.98 \pm 0.035$ & $6.47 \pm 0.186$ & 1. & -0.49 & $\ldots$ & $\cdots$ \\
\hline $21: 03: 47.19 \pm 0.20 \quad 50: 11: 27.63 \pm 0.29$ & $13.25 \pm 0.001$ & $12.62 \pm 0.001$ & $12.44 \pm 0.001$ & $12.31 \pm 0.006$ & $11.94 \pm 0.005$ & $511.61 \pm 0.015$ & $10.73 \pm 0.015$ & $8.02 \pm 0.116$ & 0.19 & -1.06 & 637 & 19. \\
\hline $21: 03: 48.42 \pm 0.3750: 14: 18.15 \pm 0.13$ & $11.08 \pm 0.011$ & $10.29 \pm 0.001$ & $9.84 \pm 0.001$ & $9.11 \pm 0.003$ & $8.68 \pm 0.003$ & $8.15 \pm 0.004$ & $7.44 \pm 0.003$ & $3.32 \pm 0.006$ & 1.97 & -0.91 & $\ldots$ & $\ldots$ \\
\hline 21:03:48.89 $\pm 0.12 \quad 50: 10: 04.72 \pm 0.54$ & $13.33 \pm 0.001$ & $12.64 \pm 0.001$ & $12.33 \pm 0.001$ & $11.96 \pm 0.005$ & $11.78 \pm 0.005$ & $511.65 \pm 0.019$ & $10.94 \pm 0.055$ & $6.20 \pm 0.043$ & 0.27 & -1.71 & 638 & 28. \\
\hline $21: 03: 49.03 \pm 0.1450: 16: 02.59 \pm 0.26$ & $12.13 \pm 0.002$ & $211.25 \pm 0.001$ & $10.62 \pm 0.001$ & $9.55 \pm 0.002$ & $9.23 \pm 0.003$ & $8.97 \pm 0.004$ & $8.56 \pm 0.016$ & $6.26 \pm 0.064$ & 0.95 & -1.72 & 639 & 6.8 \\
\hline 21:03:50.14 $\pm 0.0150: 10: 23.79 \pm 0.06$ & $13.26 \pm 0.001$ & $12.45 \pm 0.001$ & $11.95 \pm 0.001$ & $11.26 \pm 0.004$ & $10.85 \pm 0.003$ & $310.48 \pm 0.007$ & $9.85 \pm 0.010$ & $8.10 \pm 0.286$ & 0.22 & -1.24 & 640 & 86. \\
\hline 21:03:50.80 $\pm 0.08 \quad 50: 15: 33.02 \pm 0.19$ & $14.12 \pm 0.077$ & $713.35 \pm 0.011$ & $12.92 \pm 0.006$ & $12.68 \pm 0.047$ & $12.50 \pm 0.040$ & & $\cdots$ & $\cdots$ & 1.94 & $\ldots$ & $\cdots$ & $\cdots$ \\
\hline 21:03:51.50 $\pm 0.1150: 14: 32.72 \pm 0.31$ & $10.81 \pm 0.103$ & $10.18 \pm 0.006$ & $9.62 \pm 0.001$ & $8.68 \pm 0.005$ & $8.37 \pm 0.004$ & $7.99 \pm 0.016$ & $7.63 \pm 0.041$ & $3.95 \pm 0.021$ & 3.29 & -1.62 & $\ldots$ & $\ldots$ \\
\hline 21:03:52.17 $\pm 0.02 \quad 50: 14: 43.89 \pm 0.34$ & $12.40 \pm 0.005$ & $511.30 \pm 0.001$ & $10.30 \pm 0.001$ & $9.12 \pm 0.007$ & $8.58 \pm 0.006$ & $8.03 \pm 0.026$ & $7.54 \pm 0.059$ & $\cdots$ & 1.33 & -1.00 & $\cdots$ & $\cdots$ \\
\hline 21:03:52.89 $\pm 0.05 \quad 50: 14: 38.07 \pm 0.09$ & $14.42 \pm 0.004$ & $413.64 \pm 0.003$ & $13.18 \pm 0.004$ & $12.51 \pm 0.070$ & $12.06 \pm 0.078$ & $\ldots$ & $\ldots$ & $\ldots$ & 0.25 & $\ldots$ & $\ldots$ & $\ldots$ \\
\hline $21: 03: 53.32 \pm 0.31 \quad 50: 17: 49.99 \pm 0.32$ & $16.96 \pm 0.030$ & $16.20 \pm 0.018$ & $15.80 \pm 0.020$ & $14.66 \pm 0.034$ & $14.52 \pm 0.028$ & $13.88 \pm 0$ & $13.32 \pm 0$ & $\ldots$ & 0.44 & -1.21 & $\ldots$ & $\ldots$ \\
\hline 21:03:53.56 $\pm 0.31 \quad 50: 16: 00.64 \pm 0.07$ & $14.05 \pm 0.003$ & $313.24 \pm 0.002$ & $12.75 \pm 0.001$ & $12.07 \pm 0.006$ & $11.69 \pm 0.008$ & $811.51 \pm 0.0$ & 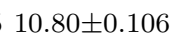 & $\ldots$ & 0.47 & -1.44 & $\ldots$ & $\cdots$ \\
\hline
\end{tabular}


Table 3-Continued

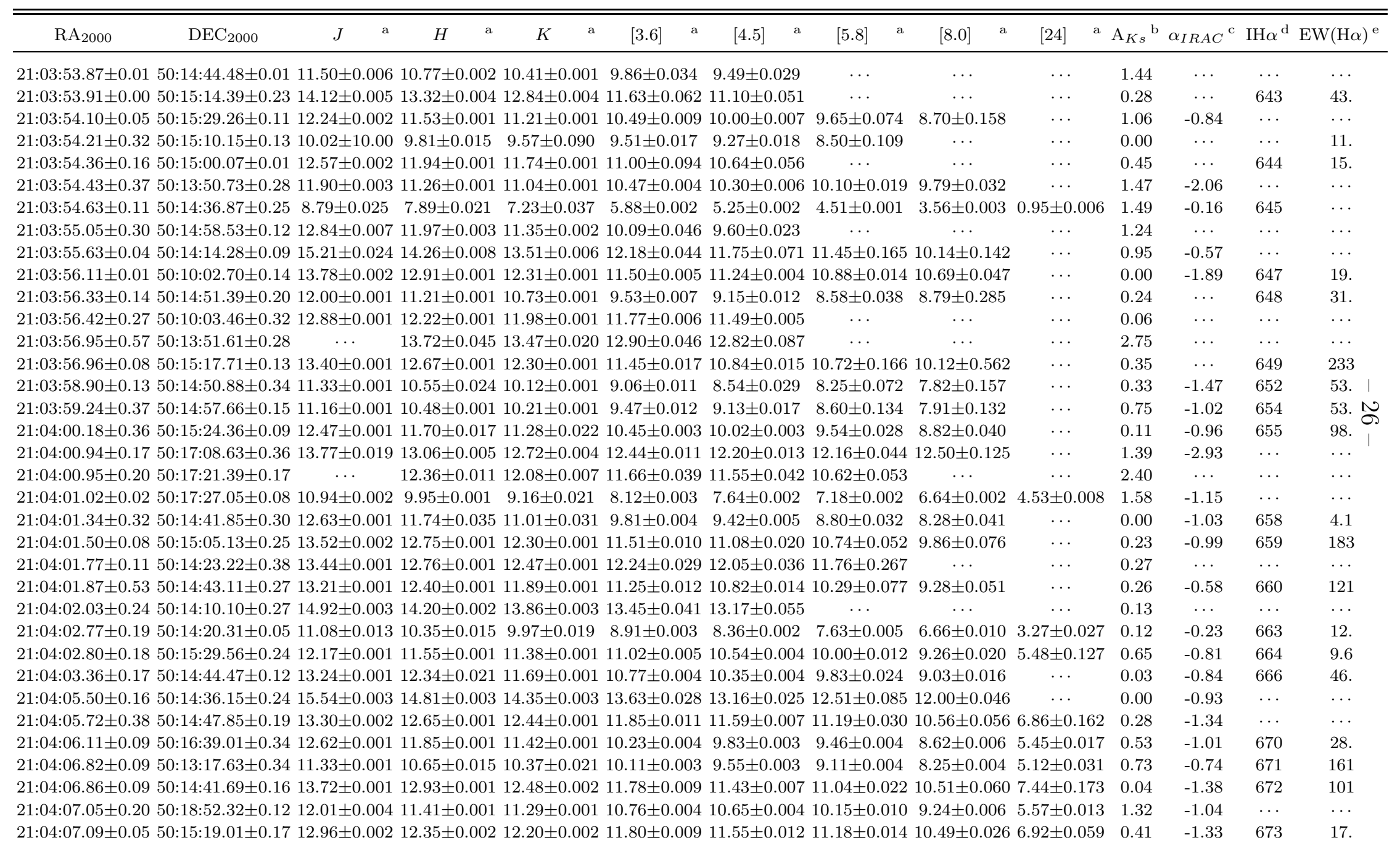


Table 3-Continued

\begin{tabular}{|c|c|c|c|c|c|c|c|c|c|c|c|c|c|c|c|c|c|}
\hline $\mathrm{RA}_{2000}$ & $\mathrm{DEC}_{2000}$ & $J$ & $\mathrm{a}$ & $H$ & $\mathrm{a}$ & $K$ & $\mathrm{a}$ & {$[3.6]$} & $\mathrm{a}$ & {$[4.5]$} & {$[5.8]$} & {$[8.0]$} & {$[24]$} & $\mathrm{A}_{K s} \mathrm{~b}$ & $\alpha_{I R A C}{ }^{\mathrm{c}}$ & $\mathrm{IH} \alpha^{\mathrm{d}}$ & $\mathrm{EW}(\mathrm{H} \alpha)^{\mathrm{e}}$ \\
\hline $21: 04: 11.23 \pm 0.03$ & $50: 10: 48.57 \pm 0.07$ & $12.03 \pm 0$ & 001 & $11.33 \pm 0$ & 017 & $11.03 \pm 0$ & 021 & $10.21 \pm 0$ & 002 & $9.94 \pm 0.003$ & $9.56 \pm 0.004$ & $8.94 \pm 0.004$ & $6.97 \pm 0.031$ & 0.27 & -1.38 & 676 & 26. \\
\hline $21: 04: 19.50 \pm 0.24$ & $50: 15: 57.86 \pm 0.22$ & $11.88 \pm 0$ & 001 & $11.00 \pm($ & & $10.40 \pm 0$ & 019 & $9.39 \pm 0$. & & $9.14 \pm 0.003$ & $8.73 \pm 0.003$ & $8.04 \pm 0.002$ & $5.21 \pm 0.010$ & 0.05 & -1.27 & 681 & 35. \\
\hline $21: 04: 20.57 \pm 0.07$ & $50: 16: 08.26 \pm 0.13$ & $13.83 \pm 0$ & 025 & $12.96 \pm 0$ & & $12.35 \pm 0$ & & $11.14 \pm 0$ & & $10.73 \pm 0.007$ & $7 \quad 10.08 \pm 0.009$ & $9.20 \pm 0.008$ & $6.37 \pm 0.027$ & 1.41 & -0.57 & $\ldots$ & $\ldots$ \\
\hline $21: 04: 28.81 \pm 0.39$ & $50: 18: 24.20 \pm 0.07$ & $13.48 \pm 0$ & 002 & $12.70 \pm 0$ & 001 & $12.25 \pm 0$ & 001 & $11.61 \pm 0$ & 004 & $11.38 \pm 0.004$ & $410.98 \pm 0.008$ & $10.14 \pm 0.008$ & $7.44 \pm 0.036$ & 0.20 & -1.15 & $\ldots$ & $\ldots$ \\
\hline
\end{tabular}

${ }^{a}$ Magnitudes are de-reddened when extinction value $\mathrm{A}_{K s}$ quoted.

${ }^{\mathrm{b}} \mathrm{A}_{K}$ determined from method outlined in $\S 5$.

${ }^{\mathrm{c}}$ Slope $\alpha$ determined from IRAC photometry

${ }^{\mathrm{d}}$ Running number from Herbig \& Dahm (2006).

${ }^{\mathrm{e}} \mathrm{EW}[\mathrm{H} \alpha]$ from Herbig \& Dahm (2006).

${ }^{\mathrm{f}}$ Slope $\alpha$ determined from [3.6], [5.8] and [24] photometry

gSlope $\alpha$ determined from K-band to longest detected wavelength 
Table 4. Objects with Mid Infrared Excesses (extragalactic contaminants removed): Transition Disks

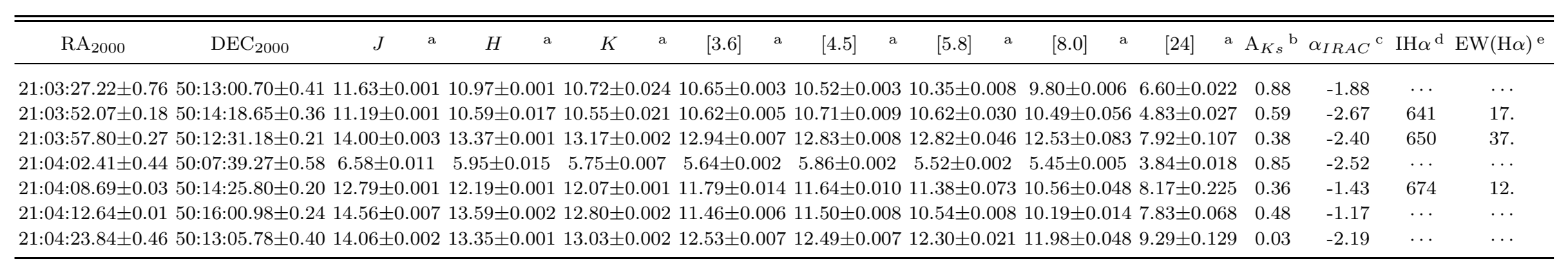

${ }^{a}$ Magnitudes are de-reddened when extinction value $\mathrm{A}_{K s}$ quoted.

${ }^{\mathrm{b}} \mathrm{A}_{K}$ determined from method outlined in $\S 5$.

${ }^{\mathrm{c}}$ Slope $\alpha$ determined from IRAC photometry

${ }^{\mathrm{d}}$ Running number from Herbig \& Dahm (2006).

${ }^{\mathrm{e}} \mathrm{EW}[\mathrm{H} \alpha]$ from Herbig \& Dahm (2006). 
Table 5. Objects without Mid Infrared Excesses (extragalactic contaminants removed): Class III

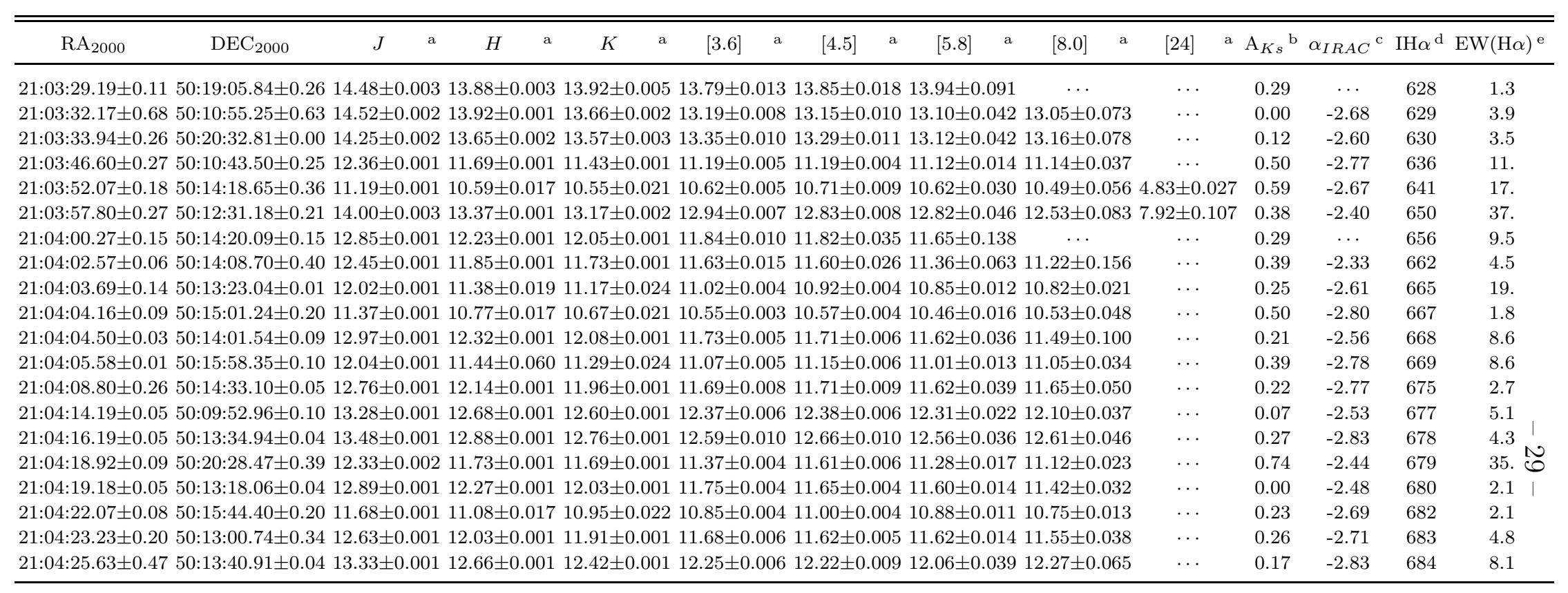

${ }^{a}$ Magnitudes are de-reddened when extinction value $\mathrm{A}_{K s}$ quoted.

${ }^{\mathrm{b}} \mathrm{A}_{K}$ determined from method outlined in $\S 5$.

${ }^{\mathrm{c}}$ Slope $\alpha$ determined from IRAC photometry

${ }^{\mathrm{d}}$ Running number from Herbig \& Dahm (2006).

e EW $[\mathrm{H} \alpha]$ from Herbig \& Dahm (2006). 
Table 6. Assumed Reddening Law from Flaherty et al. (2007).

\begin{tabular}{rc}
\hline \hline Waveband & $\mathrm{A}_{\lambda} / \mathrm{A}_{K s}$ \\
\hline $\mathrm{J}$ & 2.5 \\
$\mathrm{H}$ & 1.55 \\
$K_{s}$ & 1.0 \\
Band 1 $(3.6 \mu \mathrm{m})$ & 0.64 \\
Band 2 $(4.5 \mu \mathrm{m})$ & 0.52 \\
Band 3 $(5.8 \mu \mathrm{m})$ & 0.49 \\
Band $4(8.0 \mu \mathrm{m})$ & 0.47 \\
\hline
\end{tabular}




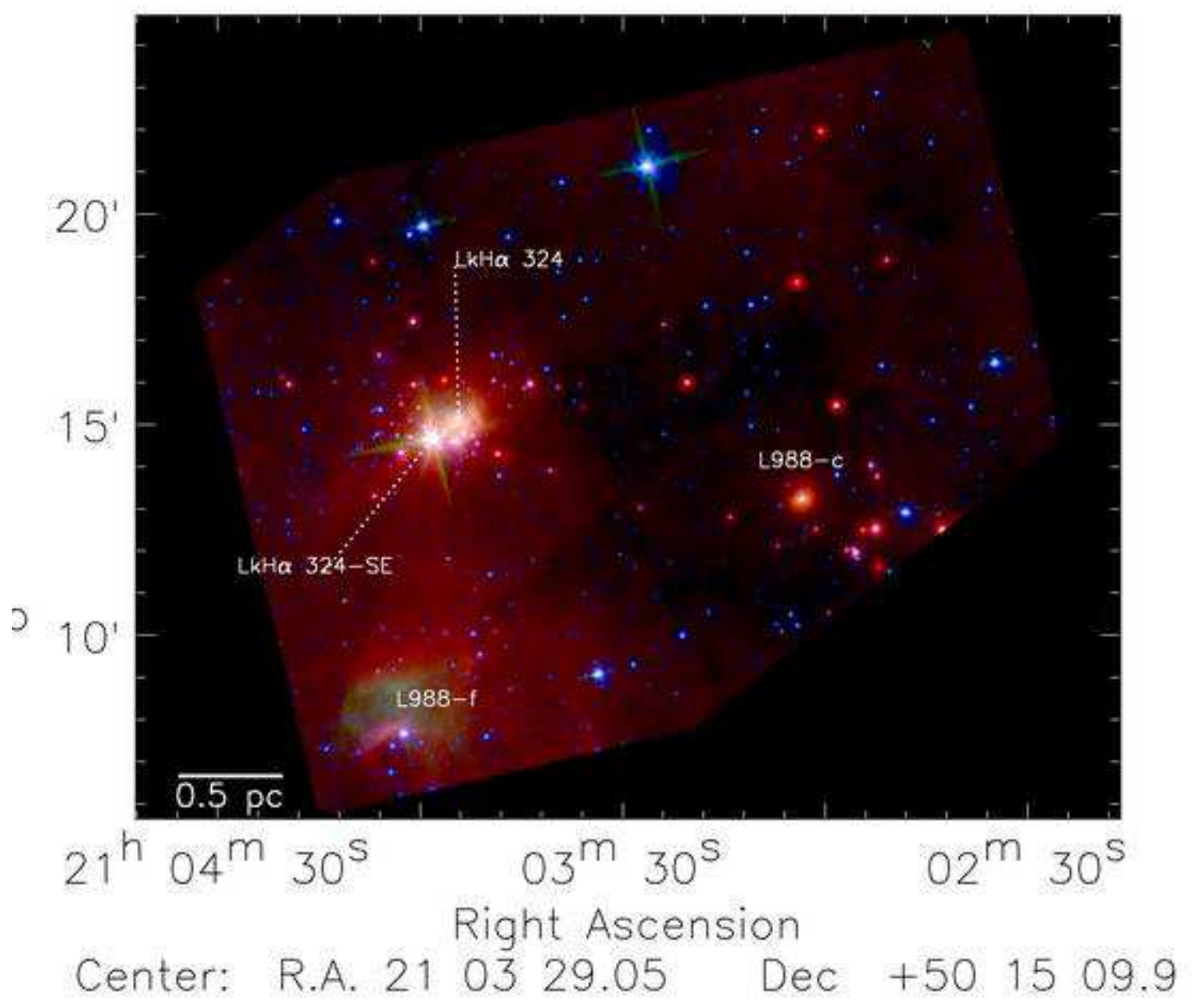

Fig. 1.- Three-color 3.6, 5.8, and $24 \mu \mathrm{m}$ image of the young asymmetric cluster L988e, including regions $\mathrm{f}$ and $\mathrm{c}$. The HAeBe stars $\mathrm{LkH} \alpha 324$ and $324 \mathrm{SE}$ are found in the L988e region. 


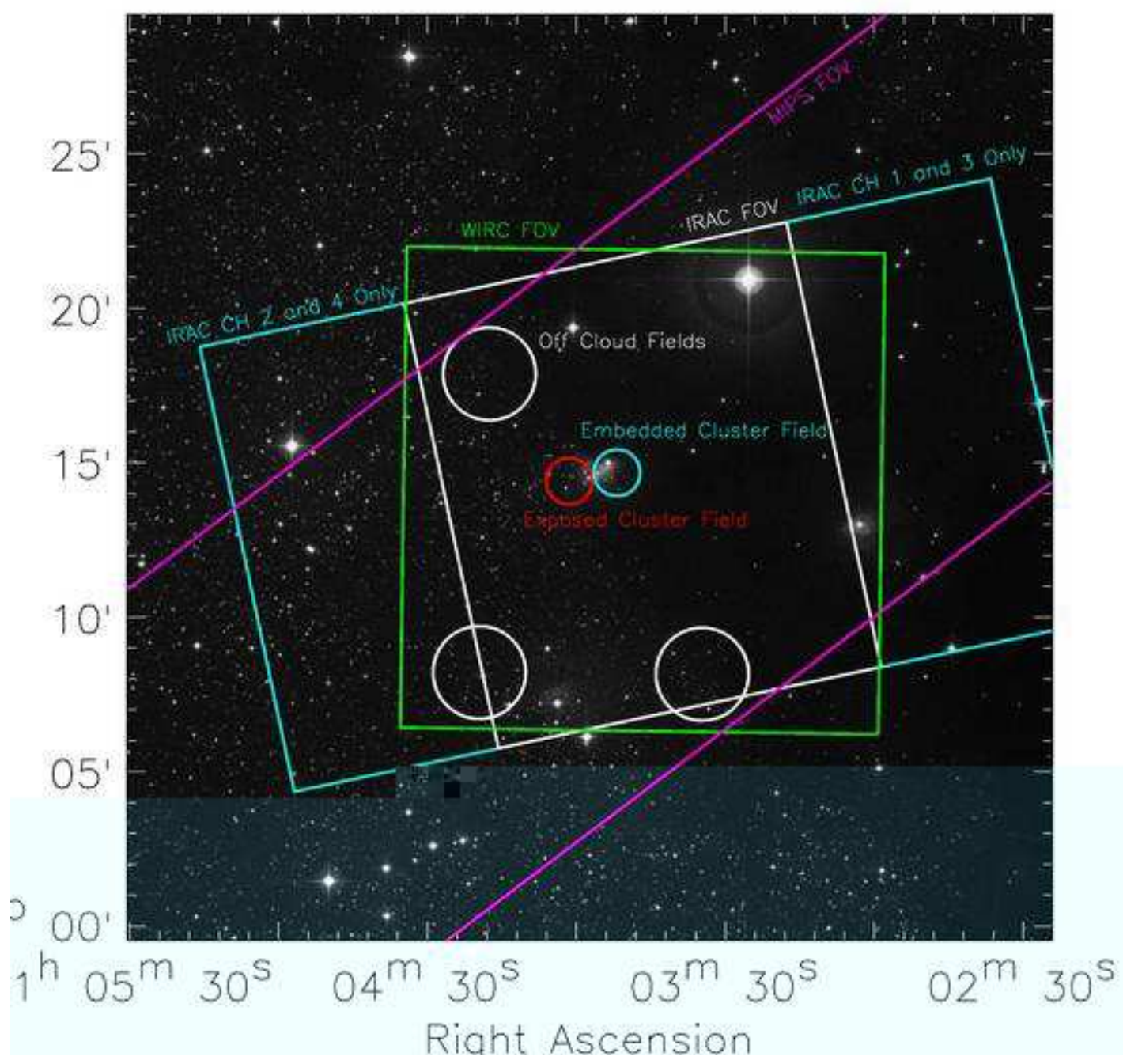

Fig. 2.- Palomar Observatory Sky Survey II V-band image with the rectangular fields of view for the data presented in this work superimposed. Additionally, the embedded (blue) and exposed (red) regions of the cluster and the off-cluster fields (white) used when determining disk fractions are shown in this figure as circles. 


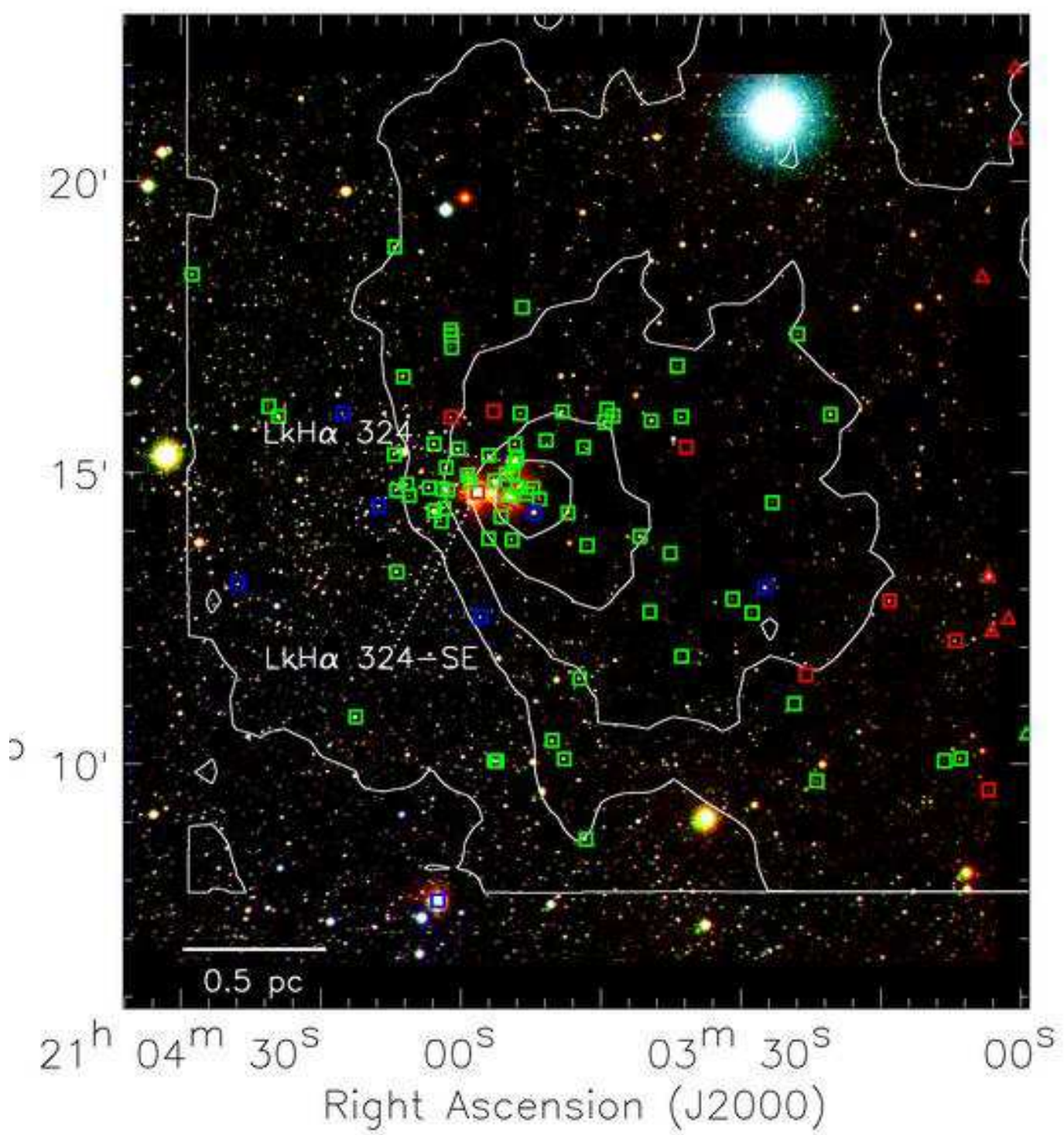

Fig. 3.- Three-color $J, H, K_{s}$, image of the L988e region, with contours of ${ }^{13} \mathrm{CO}$ emission $\left(50^{\prime \prime}\right.$ resolution) overplotted ((Ridge et al. 2003)). The ${ }^{13} \mathrm{CO}$ emission has a maximum integrated intensity of $13.75 \mathrm{~K} \mathrm{~km} \mathrm{~s}^{-1}$ and contours range from $10 \%$ to $90 \%$ of the maximum in increments of $20 \%$. Squares designate objects classified via the $(J) H, K s,[3.6],[4.5]$ methods or IRAC+MIPS methods and triangles designate objects classified via IRAC [3.6] and [4.5] and MIPS [24] photometry. Red designates identified protostars, green designates identified stars with disks, and blue designates identified stars with transition disks. Note the paucity of stars detected toward the molecular cloud in the image. 


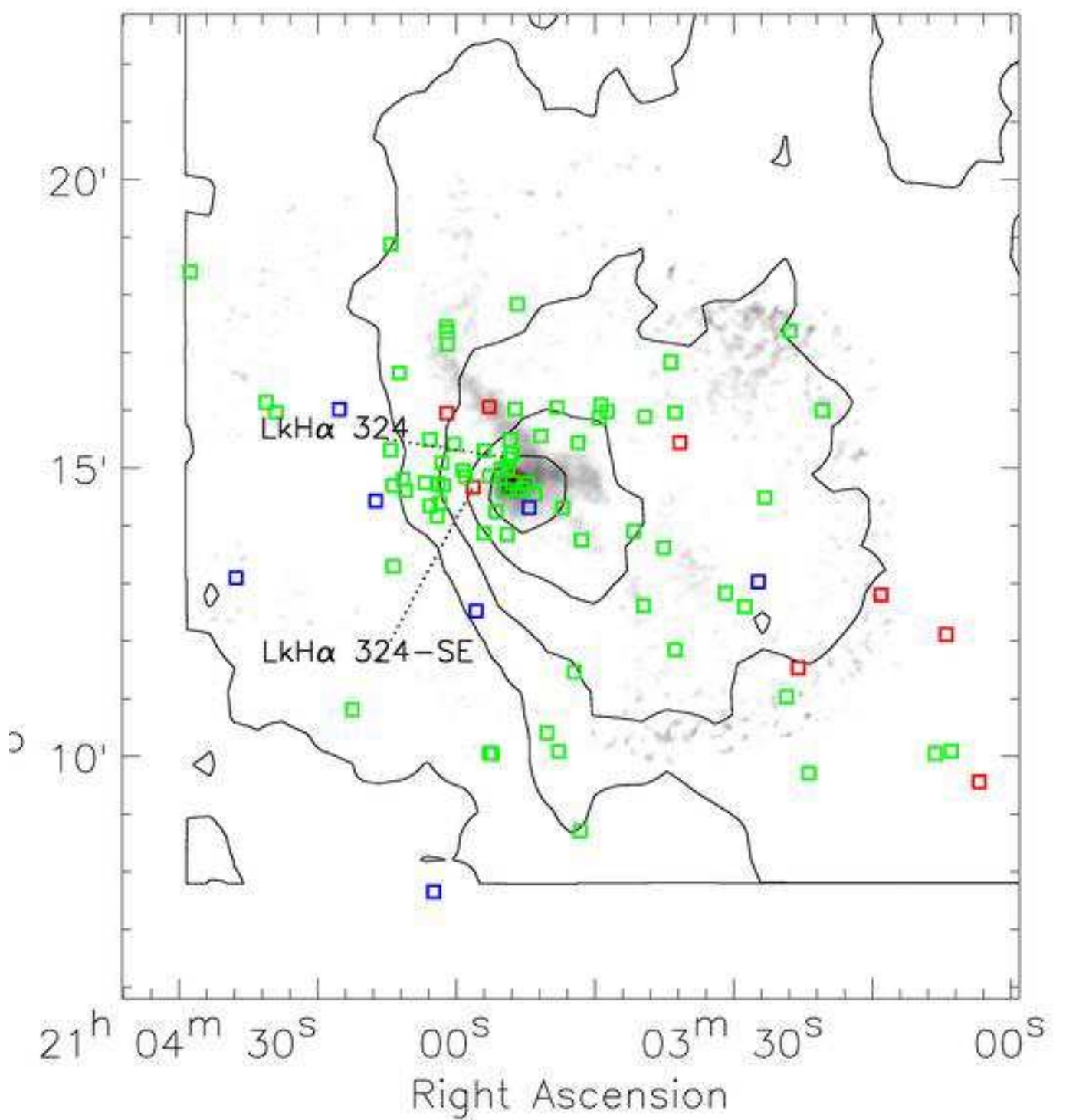

Fig. 4.- Linear grey scale SCUBA $850 \mu \mathrm{m}$ emission (15" resolution), with contours of ${ }^{13} \mathrm{CO}$ (50" resolution) overplotted, with the same contours as in Fig. 3. Red designates identified protostars, green designates identified stars with disks, and blue designates identified stars with transition disks. 


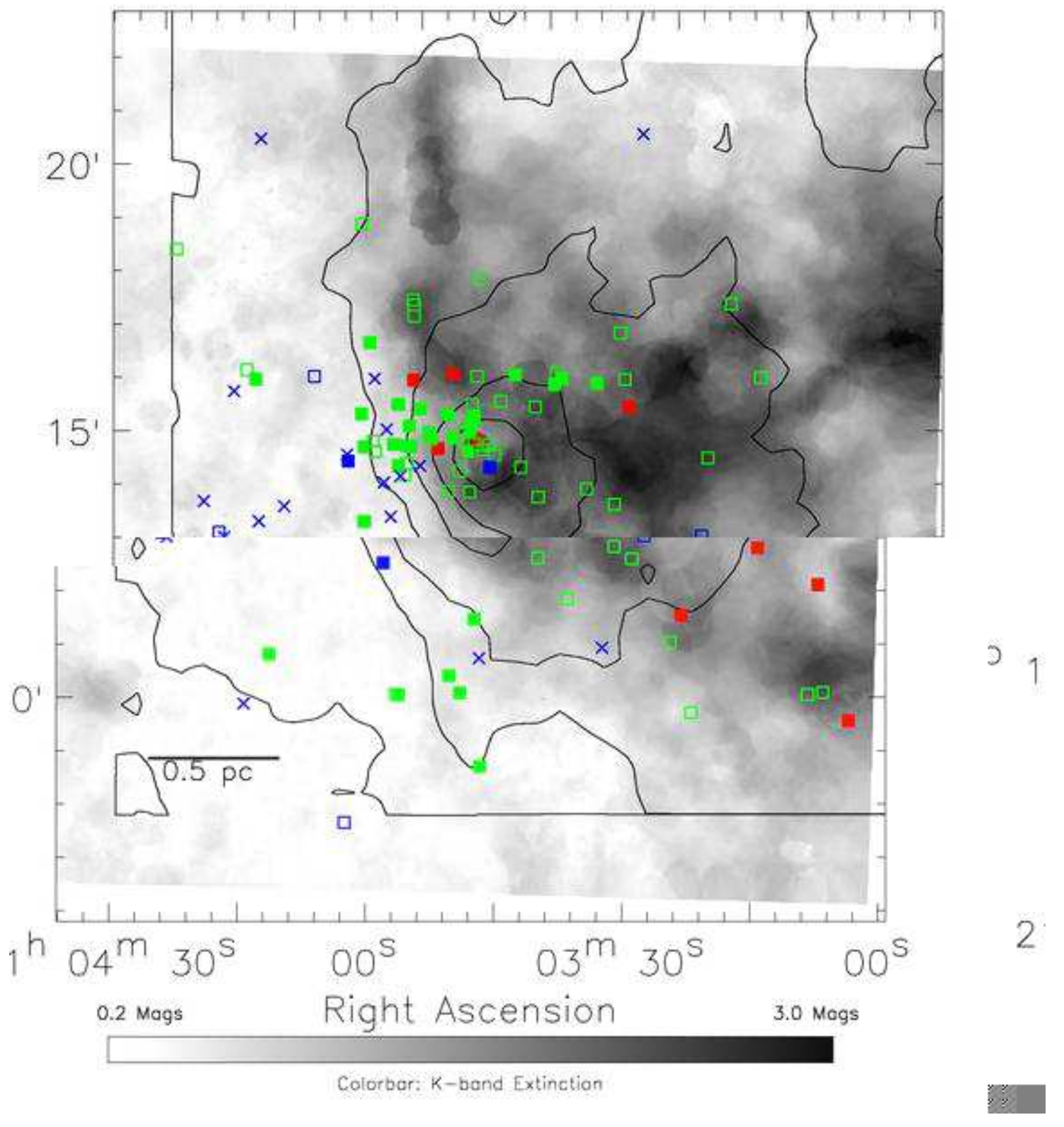

Fig. 5.- $\mathrm{A}_{K s}$ extinction map from WIRC data in grey scale, with the same ${ }^{13} \mathrm{CO}$ contours as in Fig. 3, and identified YSOs overplotted. The grey color scale extends from $\mathrm{A}_{K s}=$ 0.2 (white) to 3 (black) mag. In the region of the maximum CO intensity, SCUBA derived values are substituted for $\mathrm{A}_{K s}$. Identified YSOs are plotted with red squares denoting class Is, green squares denoting class IIs, blue squares denoting transition disk objects, and blue $\times$ 's denoting class IIIs with $\mathrm{H} \alpha$ emission. The YSO identified by filled symbols are $\mathrm{H} \alpha$ emission-line stars (Herbig \& Dahm 2006). 


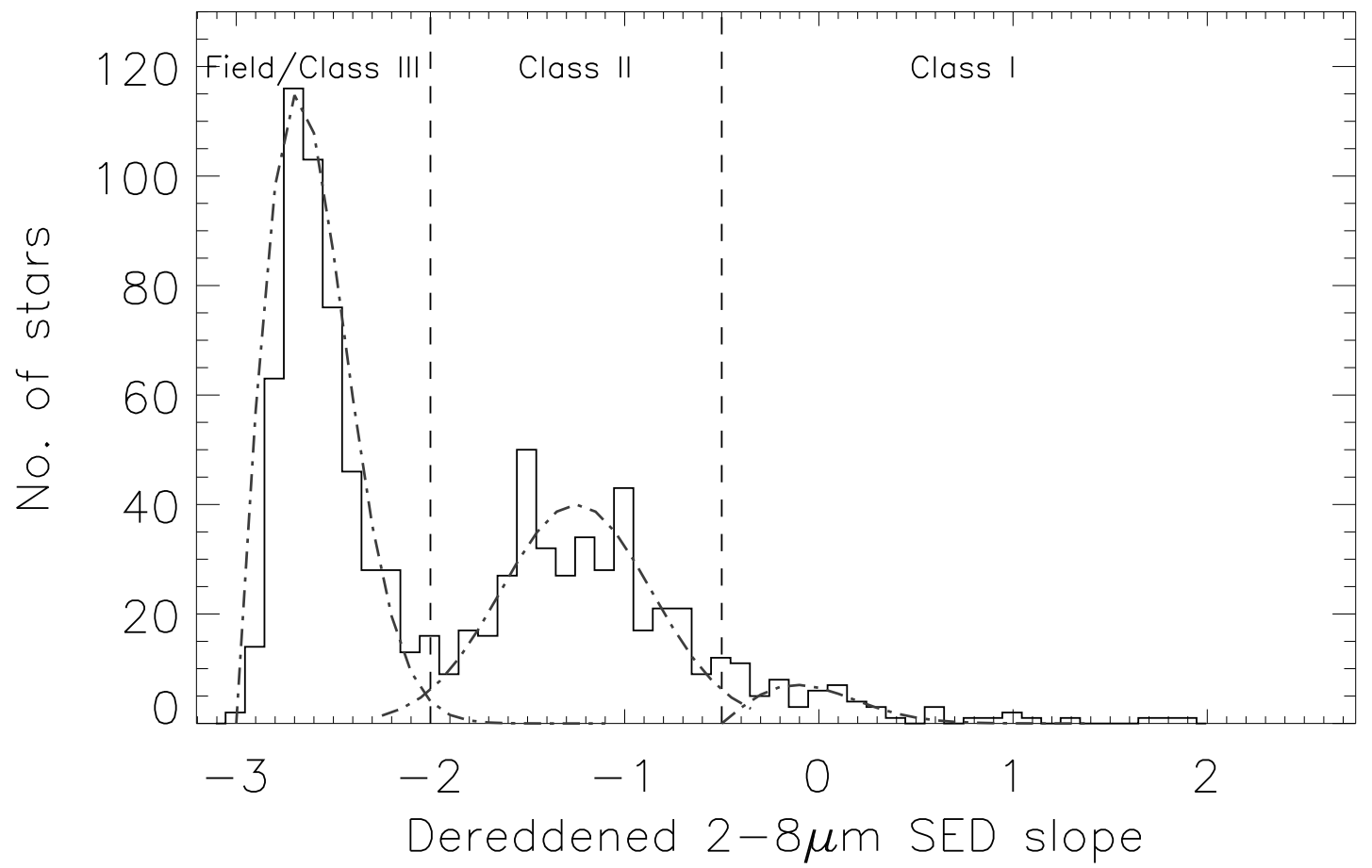

Fig. 6. - Histogram of the number of objects vs. de-reddened $2-8 \mu \mathrm{m}$ slope for six young clusters included in Gutermuth (2005). Class III/Field stars are well separated from class IIs, and class Is are defined by consideration of distribution shapes. 


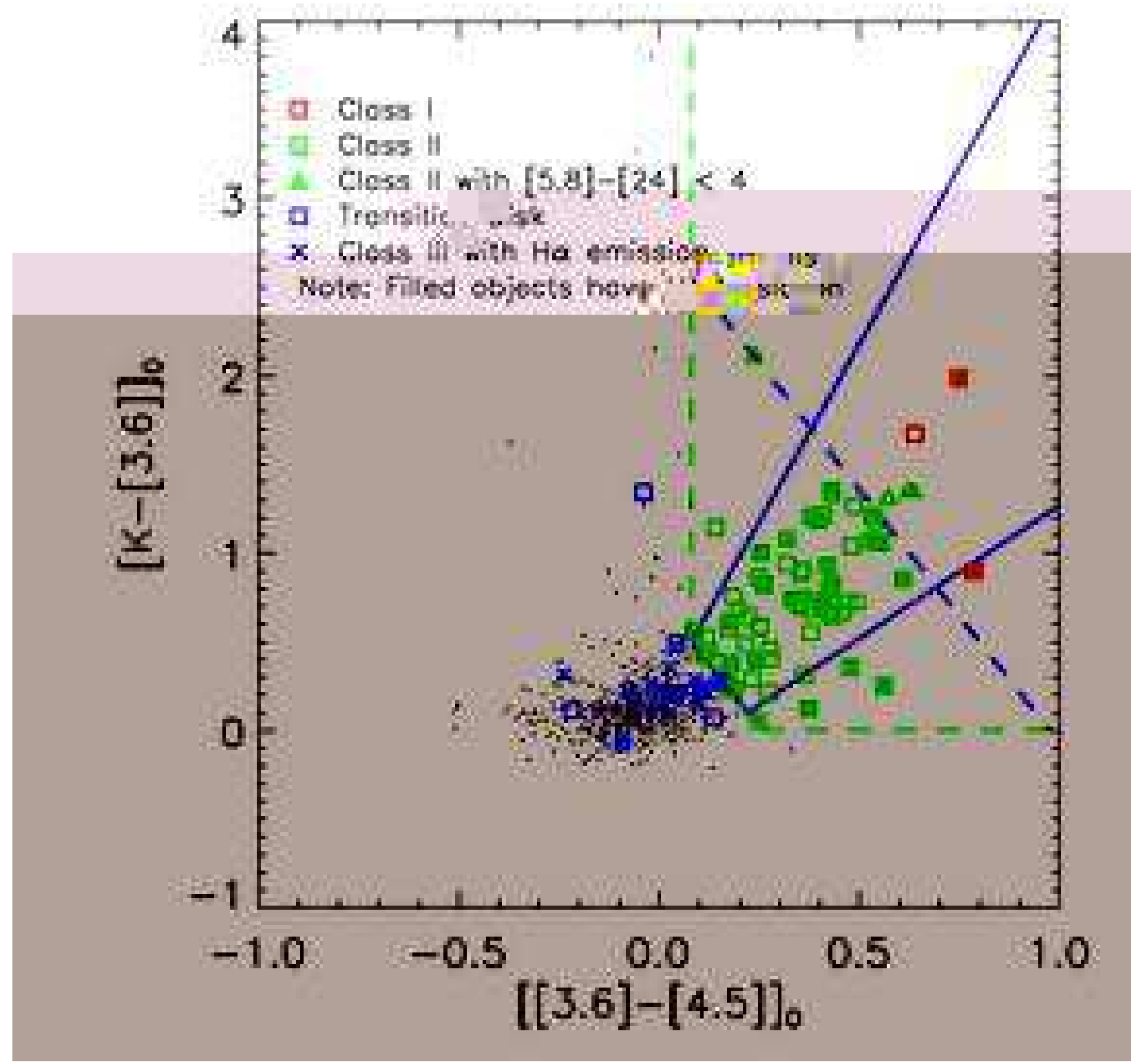

Fig. 7.- Dereddened $\left(K_{s}-[3.6]\right)_{o}$ vs. $([3.6]-[4.5])_{o}$ color-color diagram. Black dots represent either class III or field stars. A dashed blue line shows the separation between class I and II. Solid blue lines show the limits of regions clearly dominated by class I and II objects, as given by de-reddened 2-8 $\mu \mathrm{m}$ SED classifications, while the dashed green lines show the less restrictive region actually classified here, allowing for YSO variability. Triangles denote objects originally classified as class I based on dereddened $\left(K_{s}-[3.6]\right)_{o}$ vs. $([3.6]-[4.5])_{o}$ colors, but were reclassified as class II due to a $([5.8]-[24])$ color of less than 4. 


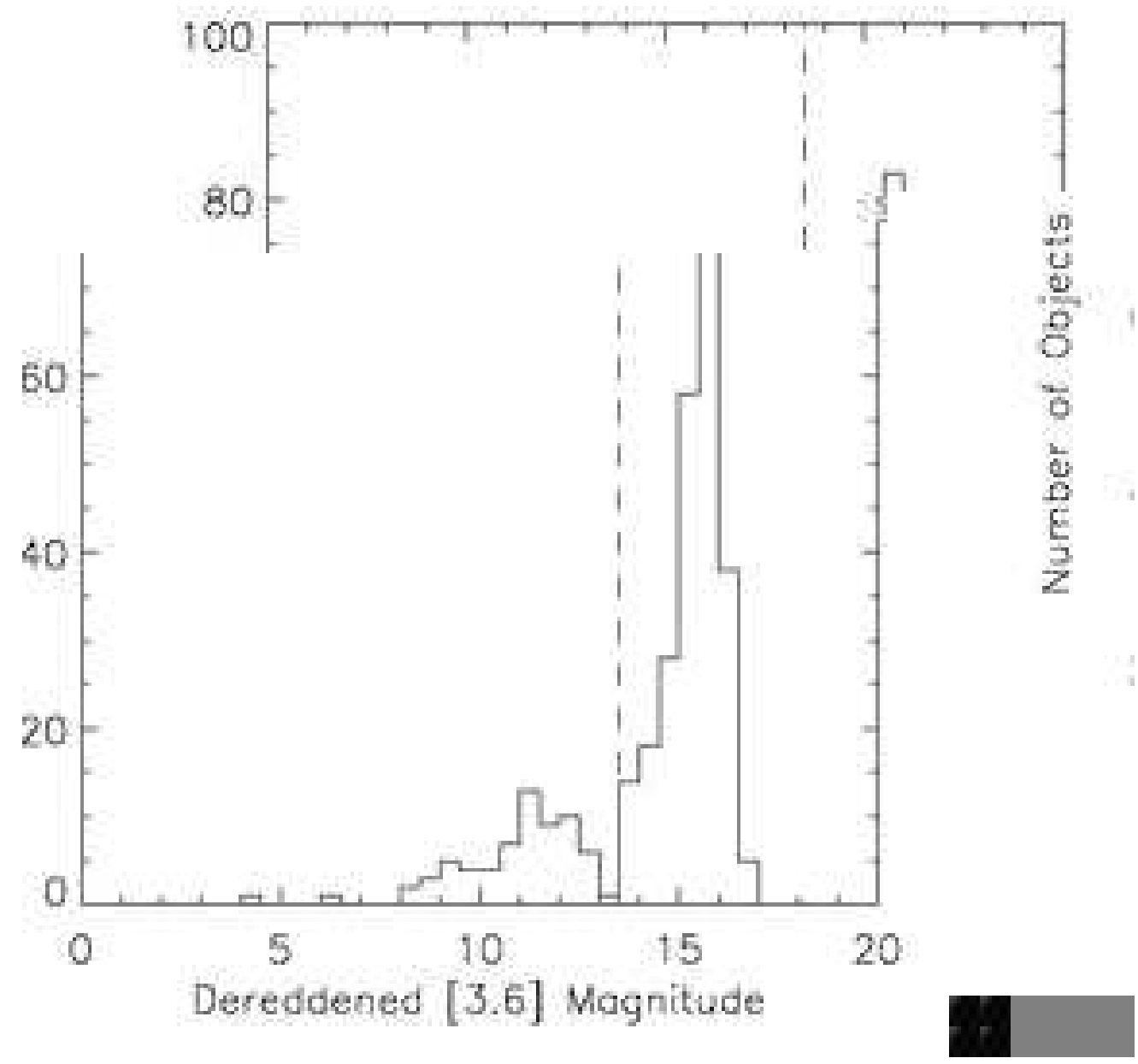

Fig. 8.- Histogram of the number of stars vs. de-reddened $3.6 \mu \mathrm{m}$ magnitudes for all objects classified as having an infrared excess from $(J) H, K_{s},[3.6],[4.5]$ photometry. Note the separation between YSOs $([3.6]<13.5)$ and extragalactic contaminates $([3.6]>13.5)$. 


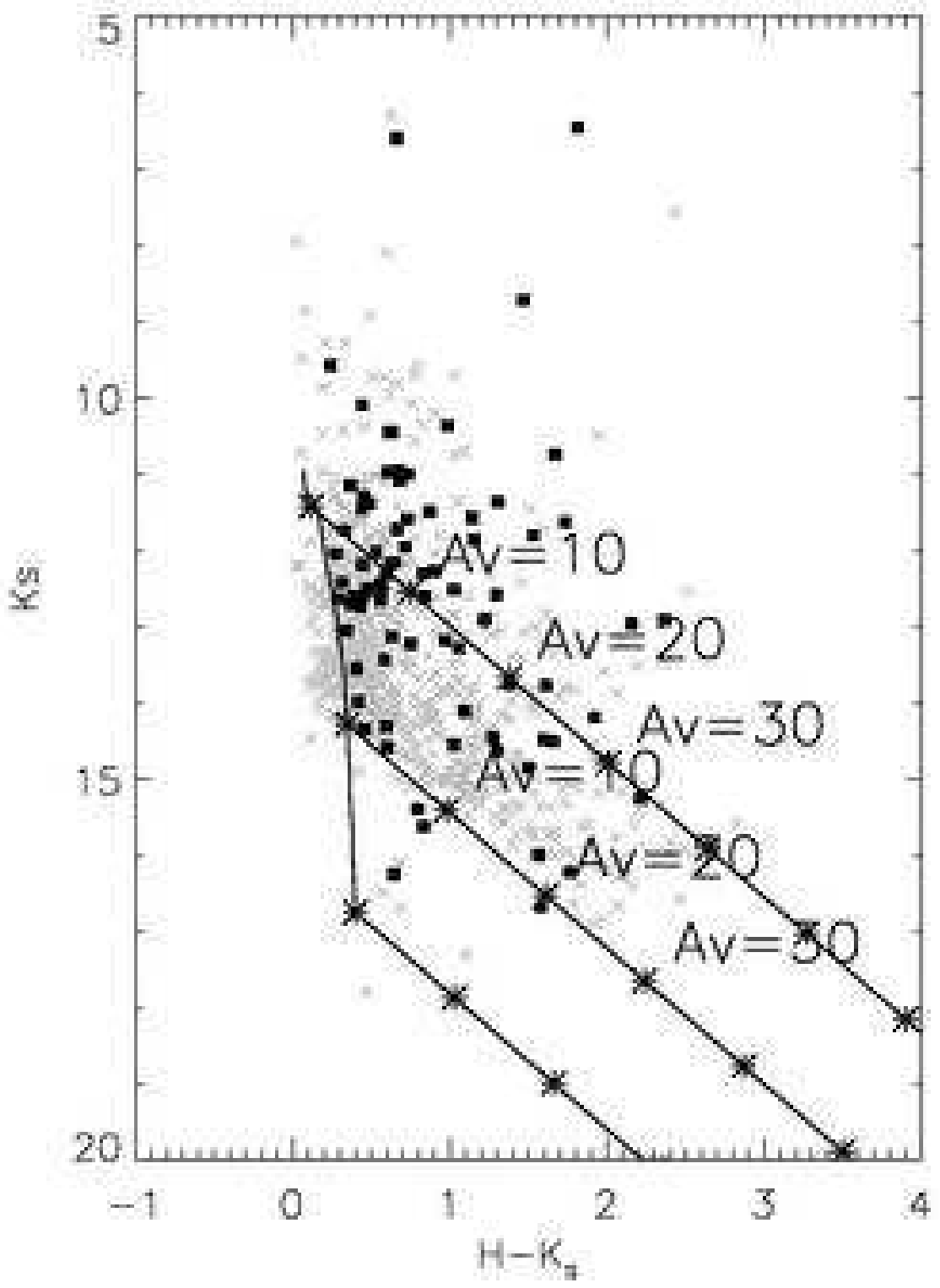

Fig. 9.- NIR Color-Magnitude diagram for cluster members (extragalactic contaminants removed) of the L988e region with the $1 \mathrm{Myr}$ isochrone of Baraffe et al. (1998) overlaid in black. Solid lines are the reddening vectors for a $1 M_{\odot}$ object (top), $0.08 M_{\odot}$ object, the Hydrogen Burning Limit (middle), and $0.025 M_{\odot}$ object (bottom). Gray ×'s represent objects classified as class III/photospheric and black squares represent objects classified as YSOs by a $J, H, K_{s},[3.6]$, [4.5] classification. 


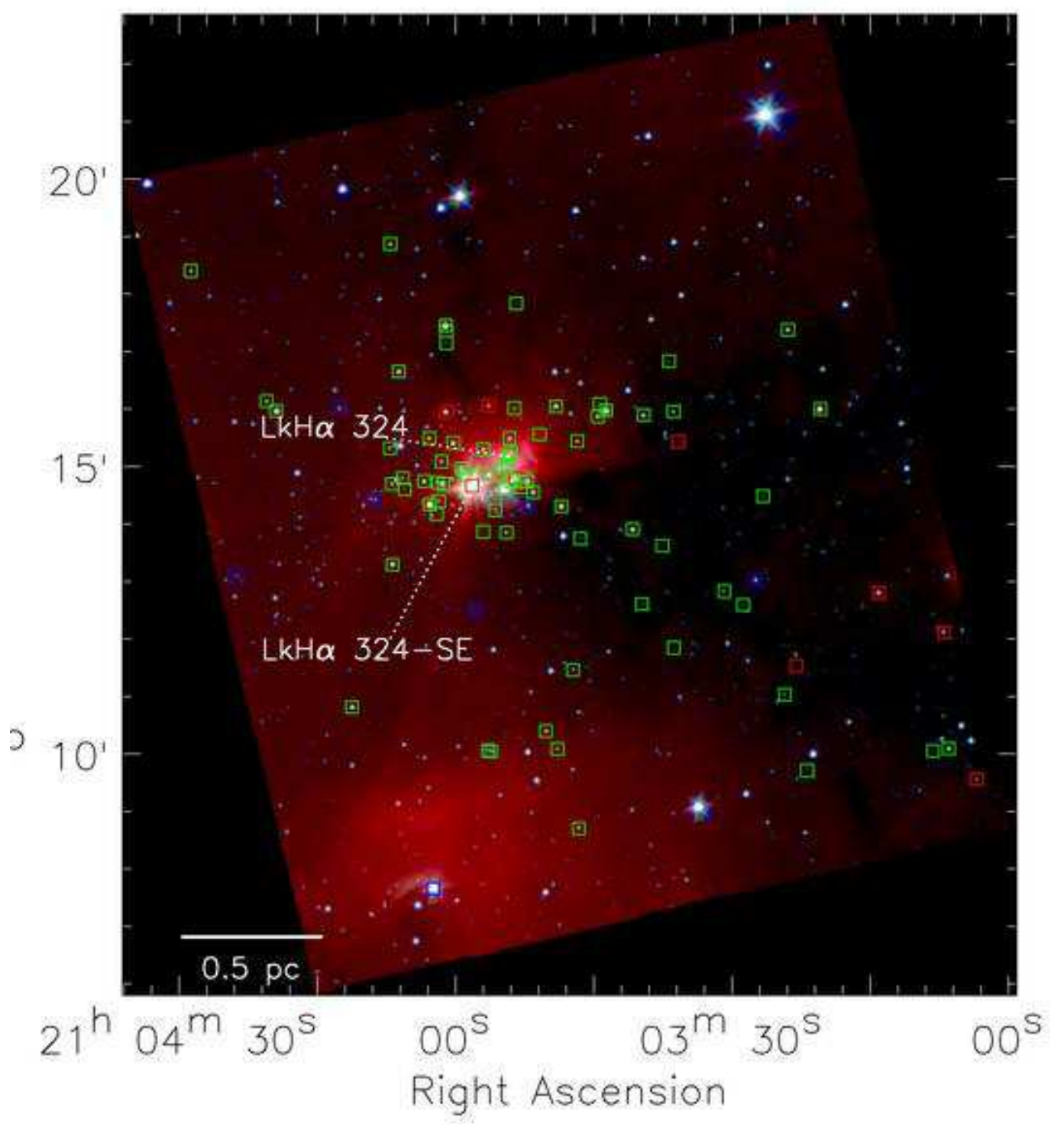

Fig. 10.- Three-color [3.6], [4.5], [8.0] image of the L988e star formation region. Overplotted on the image are stars with disks (green boxes), transition disks (blue boxes), and protostars (red boxes). 


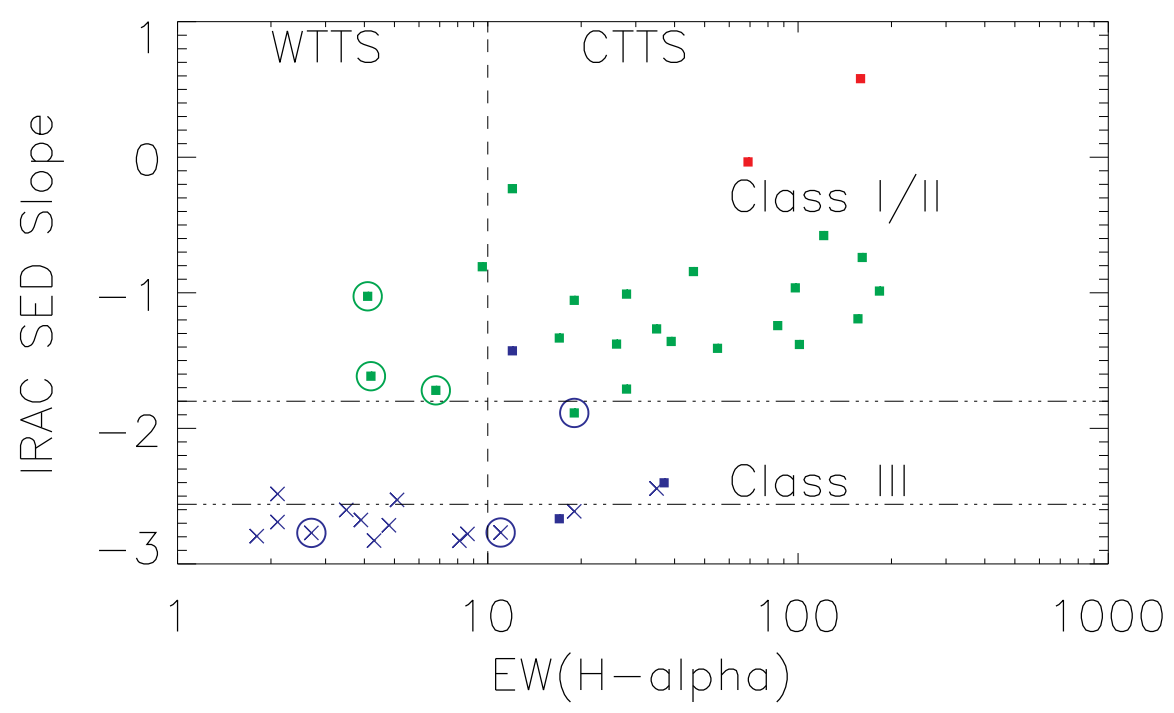

Fig. 11.- De-reddened SED slope (from $3.6 \mu \mathrm{m}$ to $8 \mu \mathrm{m}$ ) vs. EW[H $\alpha]$ in $\AA$. Here red squares represent class Is, green squares class IIs, blue squares transition disks and blue $\times$ 's class IIIs determined from $(J), H, K_{s},[3.6],[4.5]$ photometry. A vertical dashed line at $10 \AA$ illustrates the accepted single-valued equivalent width division between WTTS $(<10 \AA)$ and CTTS (>10 $\AA$ ). Circled objects are those that would yield a different CTTS/WTTS classification using the White \& Basri (2003) criterion for CTTS/WTTS classification as a function of spectral type. Green circles imply CTTS rather than WTTS as determined from the single-valued equivalent width division, and blue circles imply WTTS rather than CTTS. Two horizontal dot-dashed lines at slopes of -1.8 and -2.56 delimit the 'anemic' disk region. Sources with thick disks fall above the -1.8 line and sources without disks fall below the -2.56 line. As demonstrated by Lada et al. (2006), sources with disks (and envelopes) have stronger $\mathrm{H} \alpha$ equivalent widths. 


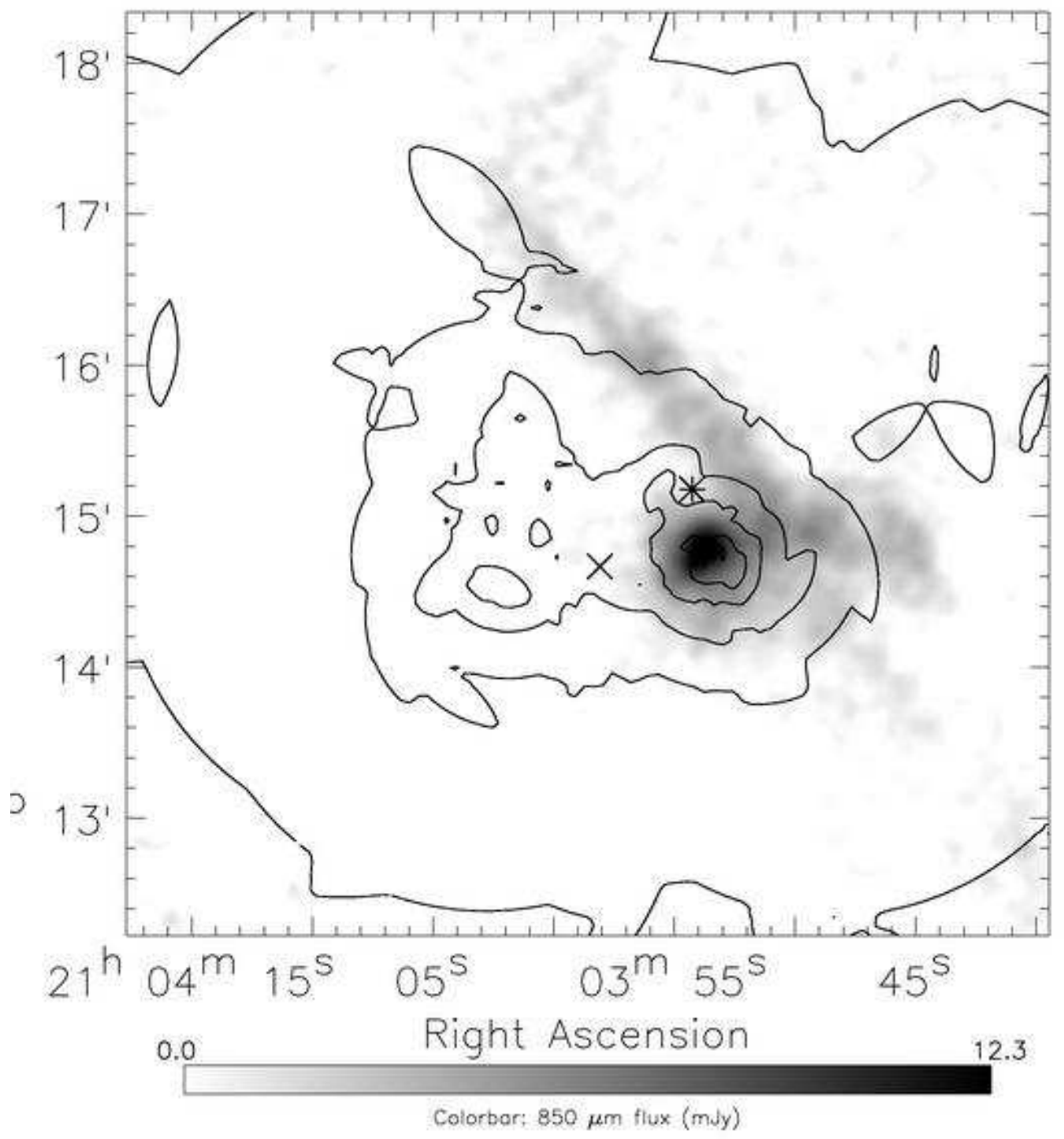

Fig. 12.- Grey scale SCUBA $850 \mu \mathrm{m}$ emission in greyscale, overplotted by contours of the surface density distributions of classified cluster members. The locations of $\mathrm{LkH} \alpha 324$ and 324SE are given by an astrisk and an $\times$, respectively. The most compact distribution, the embedded population, with a relatively high percentage of class Is, is concentrated on the dust maximum. To the east of the dust ridge, there is a second, less compact concentration, of primarily class IIs, associated with the emission-line population (Herbig \& Dahm 2006). 

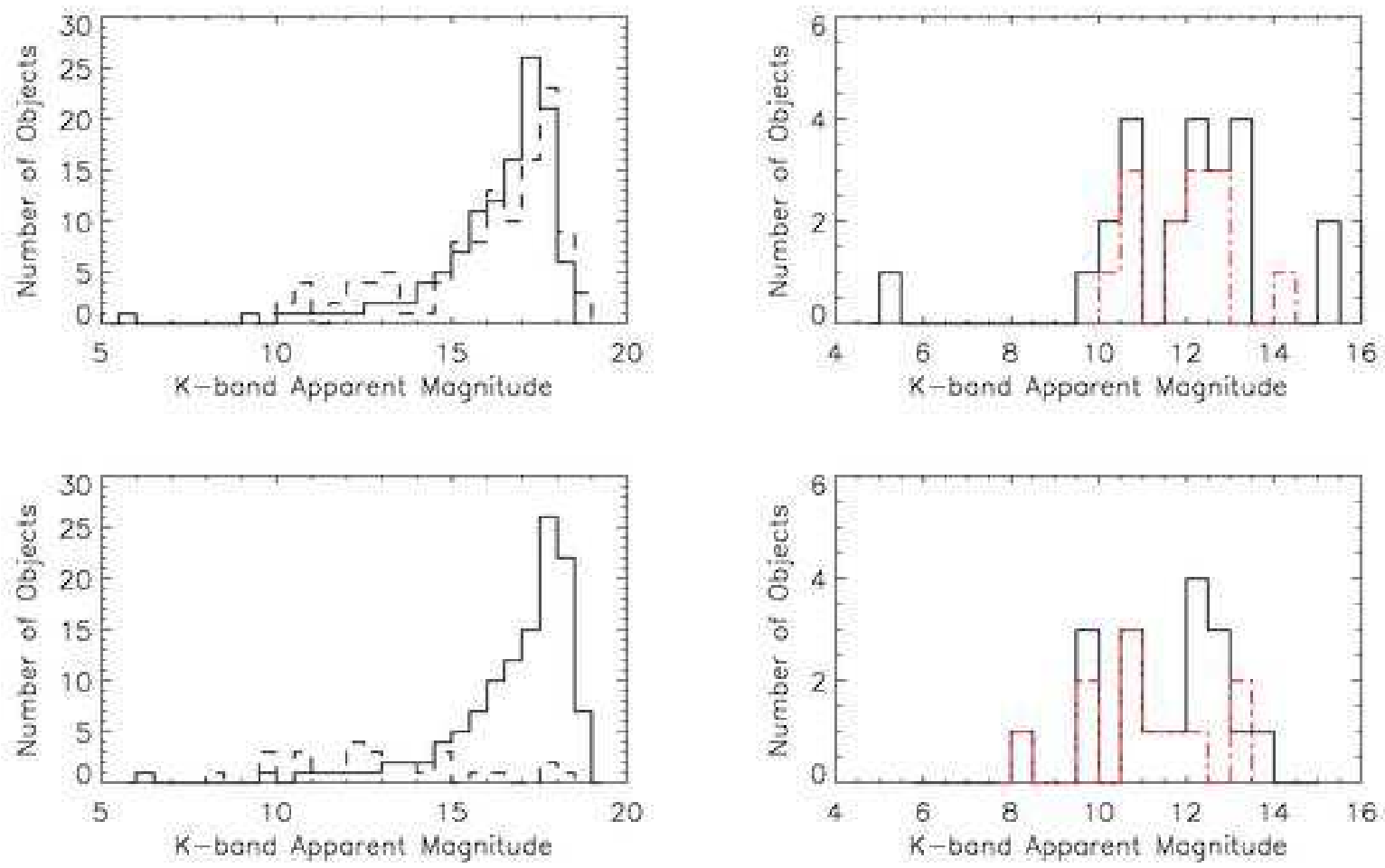

Fig. 13. - Top Left: histograms of $K_{s}$-band detections in $1.5^{\prime}$ diameter circle centered on an off-cloud region (solid line) and on the exposed cluster (dashed line). Top Right: histograms of remaining 22 objects after subtracting the off-cloud sample from the exposed cluster sample (black, solid line) and the known population with disks (red, dot-dashed line). Bottom Left: histograms of $K_{s}$-band detections in $1.5^{\prime}$ diameter circle centered on an off-cloud region (solid line) and on the embedded cluster (dashed line). Bottom Right: histograms of remaining 19 objects after subtracting the off-cloud sample from the exposed cluster sample (black, solid line) and the known population with disks (red, dot-dashed line). 